\title{
CATÁLOGO DE LOS MEGACHILIDAE DEL MEDITERRÁNEO OCCIDENTAL (HYMENOPTERA, APOIDEA). II. LITHURGINI Y MEGACHILINI ${ }^{1}$
}

\author{
C. Ornosa*, F. J. Ortiz-Sánchez** \& F. Torres***
}

\begin{abstract}
RESUMEN
El catálogo objeto de este trabajo es el segundo de una serie de tres relativa a la familia Megachilidae del territorio comprendido corológicamente en el área mediterránea occidental. Incluye los taxones que componen las tribus Lithurgini y Megachilini (subfamilias Lithurginae y Megachilinae, respectivamente), así como su distribución geográfica. Hasta el momento, la fauna de estas dos tribus está constituida por 5 géneros, 16 subgéneros y 106 especies que suponen 125 subespecies.

Palabras clave: Hymenoptera, Apoidea, Megachilidae, Lithurgini, Megachilini, catálogo, Mediterráneo occidental.
\end{abstract}

\section{ABSTRACT \\ Catalogue of Western Mediterranean Megachilidae (Hymenoptera, Apoidea). II. Lithurgini and Megachilini}

The present work is the second of a series of three that constitute a catalogue of the family Megachilidae in the western Mediterranean region. It includes the taxa of tribes Lithurgini and Megachilini (subfamilies Lithurginae and Megachilinae, respectively), their synonymies, discussion when necessary, as well as their geographic distribution. The results of our study indicate that the fauna of these tribes in the western Mediterranean is composed of 5 genera, 16 subgenera, 106 species and 125 subspecies.

Key words: Hymenoptera, Apoidea, Megachilidae, Lithurgini, Megachilini, catalogue, western Mediterranean.

\section{Introducción}

Los megaquílidos constituyen una familia de himenópteros, numerosa en especies, de distribu- ción muy amplia, prácticamente mundial en algunos de sus géneros, que abarca los reinos Holártico, Australiano y Neotropical, en las regiones biogeográficas Neártica, Paleártica, Etiópica, Oriental,

Subvencionado por el Proyecto CGL2004-04680-C10-05.

* Departamento de Zoología y Antropología Física. Facultad de Biología. Universidad Complutense. c/ José Antonio Nováis, 2. E-28040 Madrid, España. E-mail: paddy@bio.ucm.es

** Grupo de Investigación "Transferencia de I+D en el Área de Recursos Naturales". Universidad de Almería. E-04120 La Cañada de San Urbano (Almería), España. E-mail: fjortiz@ual.es

*** Departamento de Biología Animal, Ecología, Parasitología y Edafología. Universidad de Salamanca. Campus Miguel de Unamuno s/n. E-37071 Salamanca, España. E-mail: torres@usal.es 
Australiana y Neozelandesa, y aparece tanto desde zonas subárticas a desiertos como en áreas tropicales (Michener, 2000). En la región occidental del área mediterránea está representada por 5 tribus y en torno a 25 géneros, dependiendo del criterio del autor considerado (Ornosa et al., 2006).

El conocimiento que se tenía hasta ahora de los integrantes de las tribus Lithurgini y Megachilini en el ámbito mediterráneo occidental, al igual que ocurre con el resto de la familia, es deficiente. Existen datos en numerosas obras que quedan reunidos en otras más generales sobre la familia, como los catálogos de Rasmont et al. (1995), Schwarz et al. (1996), Banaszak \& Romasenko (2001) o Quaranta et al. (2004). Su estudio se había tratado de modo desigual o fragmentario, bien por haberse hecho en trabajos que incluían otros territorios, bien por centrarse meramente en la Península Ibérica o el norte de África, tal como se revisó en Ornosa et al. (2006). Merece la pena destacar la aportación específica de autores como Pérez $(1879,1890,1895$, 1897), Friese (1895, 1898, 1899, 1904, 1911, 1925), Alfken (1914, 1924, 1931, 1932, 1938a, 1938b, 1941), Alfken \& Bischoff (1933), Benoist (1924, 1934, 1935, 1940, 1950, 1969), Pasteels (1966), Tkalcu $(1967,1970,1974,1975,1988)$, van der Zanden (1977, 1983, 1986, 1988, 1989, 1998), Warncke (1980, 1981, 1986, 1988, 1992), Liongo li Enkulu (1988), Erlandsson (1991) y, en concreto, los pulicados por autores ibéricos para la Península Ibérica y Marruecos, como los de Dusmet (1906, 1915a, 1915b, 1921, 1931), Ceballos (1956, 1959), Diniz (1960a, 1960b, 1989), Pérez-Íñigo (1984), Heras \& Gayubo (1989), etc.

El objetivo fundamental de la serie que comenzó en un trabajo anterior (Ornosa et al., 2006) es clarificar cuáles son los componentes de las subfamilias, tribus, géneros y especies de megaquílidos del área mediterránea occidental y establecer su distribución, como punto de partida de la revisión de esta familia que se realiza dentro del proyecto "Fauna Ibérica". Debe recurrirse al mencionado artículo para una introducción más amplia y general sobre el estado de conocimiento y la relación pormenorizada de los antecedentes, tratamiento, autores y publicaciones globales de la familia Megachilidae en el territorio considerado. Esta segunda parte se dedica a las tribus Lithurgini y Megachilini.

\section{Material y métodos}

El presente catálogo incluye taxones no sólo ibéricos sino también del territorio mediterráneo occidental de ambas orillas del mar (sin incluir las de localidades más meridionales, netamente desérticas, de Argelia y Marruecos) y hasta Italia y Grecia por el este. Se incluyen Grecia o Italia cuando forman parte de otras distribuciones más occidentales. No obstante, no se han incluido aquéllas que desde el Mediterráneo oriental llegan hasta el entorno balcánico o las que constituyen fauna exclusiva de Grecia, la antigua Yugoslavia, de Italia o de Túnez.

Para los datos taxonómicos y de distribución, tal como se indicó anteriormente (Ornosa et al., 2006), se ha utilizado, salvo rara excepción, la bibliografía original y se han rastreado las publicaciones desde la primera cita de cada taxón hasta las más recientes de todos los ámbitos que comprende el territorio estudiado. En el texto se incluyen sólo los rangos generales de distribución y no se pormenorizan citas concretas más que en casos particulares. Del mismo modo, dada la abundante bibliografía consultada, no se especifican más referencias que aquellas que la discusión o anotaciones concretas sobre las distintas interpretaciones de ciertos taxones han requerido $\mathrm{y}$, por tanto, a pesar de haberse utilizado, no las relativas a descripciones, citas, sinonimias o datos taxonómicos, que alargarían extraordinariamente el apartado correspondiente.

La clasificación adoptada sigue, para las categorías supragenéricas, a Michener (1979), Gauld \& Bolton (1988) y Finnamore \& Michener (1993) y, tanto para los géneros como para las diversas categorías infragenéricas, a Rasmont et al. (1995), Schwarz et al. (1996) y Michener (2000). Se prescinde, por lo tanto, de las clasificaciones de Warncke (1986) y de Roig-Alsina \& Michener (1993). En cuanto a la nomenclatura supragenérica, se ha seguido a Michener (1986, 2000). Puntualizaciones precisas y específicas a este respecto se van haciendo a lo largo del texto.

Por otro lado, aunque según el criterio de Michener (2000), basado en McGinley \& Rozen (1987) y respaldado, por ejemplo, por Engel (2001, 2002, 2004), la subfamilia Fideliinae estaría incluida dentro de los megaquílidos, no se consigna ninguna especie, pues las más próximas geográficamente, si bien norteafricanas occidentales, no encajan con la distribución corológica mediterránea occidental. Pararhophites quadratus Friese, 1898 (tribu Pararhophitini), distribuida en Egipto, ha sido citada de Tan Tan, en la vertiente atlántica marroquí, frente a las Islas Canarias (Warncke, 1979), y Fidelia ulrikei Warncke, 1980 (tribu Fideliini), descrita de 
Tansikht, una localidad unos $30 \mathrm{~km}$ al este de Agdz (Marruecos), sólo ha vuelto a ser citada en lugares en torno al valle del Draa (Warncke, 1980; Patiny, com. pers.), ambiente puramente sahariano.

Asimismo, en relación con la posición sistemática de las dos tribus objeto del presente trabajo, y aunque la decisión adoptada no afecta a la naturaleza del estudio ni a los resultados obtenidos, la familia Megachilidae contendría, aparte de la subfamilia Fideliinae, otras dos (Lithurginae y Megachilinae). En Lithurginae estaría encuadrada la tribu Lithurgini y, en Megachilinae, las demás, incluida Megachilini. Esta concepción había sido modificada por Michener (2000), aunque él mismo la reconocía como válida anteriormente (Roig-Alsina \& Michener, 1993); sin embargo, parece no haber obtenido el respaldo de los especialistas, ya que, sólo un año después, Engel (2001) devolvía a los Lithurgini a su rango anterior, como subfamilia independiente del resto de los Megachilinae, porque poseen características propias que los separan claramente de los demás. No obstante, futuros estudios filogenéticos deberían tender a esclarecer la situación.

Igualmente, en cuanto a la consideración genérica, y hasta que posteriores investigaciones complementarias apoyen una determinada visión, aparte del género cleptoparásito Coelioxys Latreille, 1809, se contempla con este estatus a Creightonella Cockerell, 1908, Chalicodoma Lepeletier, 1841 y Megachile Latreille, 1802, que, por ejemplo, Michener (2000) vuelve a reunir como subgéneros de Megachile. La principal razón es que poseen caracteres distintivos propios y presentan áreas de diversidad con unos números de taxones lo suficientemente altos como para mantenerlos con estatus genérico.

De igual modo que en publicaciones anteriores (Ornosa y Ortiz-Sánchez, 2004; Ornosa et al., 2006), con el término latino auctt., que aparece en el listado tras el nombre de varios taxones, se alude a denominaciones $\mathrm{y} / \mathrm{o}$ combinaciones empleadas por numerosos autores en un sentido diferente al establecido por el autor original. Igualmente, se emplea el término partim pues, aunque las sinonimias no lo son en parte, la diferencia entre sexos en apoideos ha hecho que la confusión en ocasiones sólo haya sido parcial, por lo que, sólo a título informativo, se señala.

Por último, hay que señalar que, dadas las dificultades generadas por la falta de especificación de las localidades y las dudas sobre la real ubicación de muchas de las citas de Pérez (1895), bajo el genérico "Barbarie", se incluye esa cita original de ese modo en cada caso y se remite al lector para una más pormenorizada explicación a la introducción en Ornosa et al. (2006).

\section{Resultados}

A partir de los antecedentes bibliográficos, las tribus Lithurgini y Megachilini están representadas en la fauna mediterránea occidental por 5 géneros, 16 subgéneros, 106 especies y 125 subespecies.

\section{Tribu Lithurgini}

Género Lithurgus Berthold, 1827

Lithurge Latreille, 1825. Fam. nat. Règne Anim.: 463. No disponible, vernáculo

ESPECIE TIPO: Andrena cornuta Fabricius, 1787, por monotipia Lithurgus Berthold, 1827. Latreille's nat. Fam. Thier.: 467 ESPECIE TIPO: Andrena cornuta Fabricius, 1787, por monotipia Liturgus Ashmead, 1899. Trans. Amer. Ent. Soc. 26: 77 (non Schoenherr, 1844), grafía posterior incorrecta

Distribución geográfica: casi todo el mundo, en zonas tropicales y cálidas a moderadamente templadas, excepto el trópico húmedo de América, donde es más escaso. De los dos subgéneros actualmente reconocidos, sólo uno vive en el territorio considerado; el otro es americano (Michener, 2000).

\section{Subgénero Lithurgus Berthold, 1827}

Se reparte por Eurasia, África y Australia, así como sus islas adyacentes; existe una especie en Brasil, Lithurgus (Lithurgus) huberi Ducke, 1907, seguramente introducida (Michener, 2000). Otra, Lithurgus (Lithurgus) chrysurus Fonscolombe, 1834, muy común en nuestras latitudes, fue citada en Nueva Jersey también como introducida (Roberts, 1978), aunque posiblemente haya desaparecido ya de allí (Michener, 2000). Existen en torno a 15 especies de este subgénero, 7 de ellas paleárticas (van der Zanden, 1986).

Lithurgus (Lithurgus) cephalotes van der Zanden, 1977

Lithurge cephalotes van der Zanden, 1977. Bull. Rech. Agron. Gembloux, 12(4): 358

Especie endémica de Marruecos.

Lithurgus (Lithurgus) chrysurus Fonscolombe, 1834

Lithurgus chrysurus Fonscolombe, 1834. Ann. Soc. Entomol. France, 3: 220 
Lithurgus analis Lepeletier, 1841. Hist. Nat. Insectes Hyménopt., 2: 347

Lithurgus haemorroidalis Lepeletier, 1841. Hist. Nat. Insectes Hyménopt., 2: 346

Lithurgus chrysurus var. siculus Pérez, 1897. Compt. rend. Soc. Linn. Bordeaux, 52: 65

Lithurgus sublaevis Pérez, 1897. Compt. rend. Soc. Linn. Bordeaux, 52: $66^{2}$

Especie paleártica occidental, distribuida desde la Península Ibérica y Marruecos, hasta Irán y el Cáucaso y, por el norte, hasta Austria. Fue introducida en América, como se indica más arriba.

Lithurgus (Lithurgus) cornutus (Fabricius, 1787)

Andrena cornuta Fabricius, 1787. Mant. Ins., 1: 298

Especie repartida por toda Europa y la cuenca mediterránea, llegando al Cáucaso e Irán. Presenta tres subespecies, de las que dos se reparten por el área mediterránea occidental.

Lithurgus (Lithurgus) cornutus cornutus (Fabricius, 1787)

Andrena cornuta Fabricius, 1787. Mant. Ins., 1: 298

Lithurgus umbraculatus Lepeletier, 1841. Hist. Nat. Insectes Hyménopt., 2: 345

Lithurgus cornutus var. obscurus Cockerell, 1931. Ann. Mag. Nat. Hist., 10(7): 203

Subespecie de África noroccidental citada de "Barbarie" y Marruecos.

Lithurgus (Lithurgus) cornutus fuscipennis Lepeletier, 1841

Lithurgus fuscipennis Lepeletier, 1841. Hist. Nat. Insectes Hyménopt., 2: 347

Lithurgus nasutus Dufour, 1849. Ann. Sci. nat. sér. 3, 11: 96

Megachile monoceros Eversmann, 1852. Bull. Soc. Imp. Nat. Moscou, 25: 67

Megachile dohrni Radoszkowski, 1862. Stett. Entomol. Ztg., 23: 271

Subespecie circunmediterránea ausente del África noroccidental.

Lithurgus (Lithurgus) tibialis Morawitz, 1875

Lithurgus tibialis Morawitz, 1875. En: Fedtschenko, Izv. Imp. Obsestva. Lûbit. Estestv. Antropol. Etnogr., 19: 103

Pseudosmia tibiodentata Radoszkowski, 1888. Horae Soc. Entomol. Ross., 22: 340

Distribución amplia, que comprende desde España y Portugal, Italia, Grecia, Chipre, Palestina, Turquía e Irán, hasta Pakistán y el Turquestán.

2 Ver Ortiz-Sánchez et al. (2007) en relación con esta sinonimia.

\section{Tribu Megachilini}

Género Creightonella Cockerell, $1908^{3}$

Megachile (Creightonella) Cockerell, 1908. Entomologist, 41: 146 ESPECIE TIPO: Megachile mitimia Cockerell, $1908=$ Megachile cognata Smith, 1853, por designación original

Creightoniella Pasteels, 1965. Ann. Mus. R. Afriq. Centr., Sci. Zool., 137: 10, enmienda injustificada

Distribuido desde el sur de Europa hasta Suráfrica y, por el este, a través de Asia, hasta China, Taiwan, las Filipinas, Indonesia y las Islas Solomon; existe también un registro en el norte de Australia (Michener, 2000). El número de especies se sitúa en torno a 50, de las que sólo una vive en toda el área mediterránea.

Subgénero Metamegachile Tkalcủ, 1967

Megachile (Metamegachile) Tkalců, 1967. Acta ent. Bohemoslov., 64: 102

ESPECIE TIPO: Anthophora albisecta Klug, 1817, por designación original

Distribuido por Europa central y meridional, norte de África, Turquestán, Asia Menor y Central.

Creightonella (Metamegachile) albisecta (Klug, 1817)

Anthophora albisecta Klug, 1817. En: Germar, Reise nach Dalmat., 2: 266

Megachile sericans Fonscolombe, 1832. Magas. zool., 1: 50

Megachile caucasica Lepeletier, 1841. Hist. Nat. Insectes Hyménopt., 2: 330

Megachile dufouri Lepeletier, 1841. Hist. Nat. Insectes Hyménopt., 2: 337

Megachile odontura Smith, 1849. Zoologist, 7: 58

Megachile carinulata Costa, 1882. Rendic. Accad. Sc. fis. mat. Napoli, 21: 10, 198

Megachile gerszabiensis Radoszkowski, 1886. Horae Soc. Entomol. Ross., 20 [1885-1887]: 11

Megachile germabensis Radoszkowski, 1893. Horae Soc. Entomol. Ross., 27 [1892-1893]: 43

Distribuida ampliamente por Europa meridional y el norte de África, desde la Península Ibérica y Marruecos hasta el Asia central.

Creightonella (Metamegachile) arabica (Friese, 1901)

Megachile arabica Friese, 1901. Z. syst. Hymenopt. Dipterol. 1: 71

Distribuida por Marruecos, Egipto, Arabia y Sudán.

3 Michener (2000) consideraba Megachile como un único género y en él tres grupos de subgéneros, entre los que se encontrarían Creightonella y Chalicodoma. 
Género Chalicodoma Lepeletier, $1841^{4}$

Chalicodoma Lepeletier, 1841. Hist. Nat. Insectes Hyménopt., 2: 309

ESPECIE TIPO: Apis muraria Olivier, 1789 (como Xylocopa muraria Fabricius, 1804), (non Retzius, 1783) = Apis parietina Geoffroy, 1785, por designación de Girard, 1879

Este género, en su concepto clásico y no en el sentido de Michener (2000), como subgénero de Megachile, habita Centroeuropa, es abundante en el ámbito mediterráneo, desde las Canarias hasta Asia suroccidental, y llega por el este a Mongolia, China y Pakistán y, por el sur, a toda África. No se sigue en el texto el criterio de Michener de degradar el género Chalicodoma a subgénero y, por lo tanto, se mantienen como subgéneros los tradicionalmente así considerados y no como sinónimos del subgénero Chalicodoma sensu Michener. Hay, al menos, 11 especies paleárticas (Michener, 2000).

\section{Subgénero Allochalicodoma Tkalcủ, $1970^{5}$}

Chalicodoma (Allochalicodoma) Tkalců, 1970. Acta Mus. Morav., 54 (Suppl.) [1969]: 359

EsPECIE TIPO: Chalicodoma lefebvrei Lepeletier, 1841, por designación original

Megachile (Heteromegachile) Rebmann, 1970. Nachr. Bayer. Entomol., 19(3): 41

ESPECIE TIPO: Chalicodoma lefebvrei Lepeletier, 1841, por designación original

Subgénero distribuido por el sur de la región paleártica occidental y la región etiópica.

Chalicodoma (Allochalicodoma) albocristata ${ }^{6}$ (Smith, 1853)

Megachile albocristata Smith, 1853. Cat. Hymenopt. Insects Collect. Br. Mus., 1: 151

Megachile serrata Smith, 1853. Cat. Hymenopt. Insects Collect. Br. Mus., 1: 152

Chalicodoma (Chalicodoma) lefebvrei var. tristis Friese, 1898. Term. Füz., 21: 201

Especie mediterránea occidental, distribuida por el sur de Europa, desde Italia a la Península Balcánica, más Córcega y Sicilia.

Chalicodoma (Allochalicodoma) lefebvrei Lepeletier, 1841

Chalicodoma lefebvrei Lepeletier, 1841. Hist. Nat. Insectes Hyménopt., 2: 332

${ }^{4}$ Véase la nota al pie número 3.

5 A pesar de que en el volumen correspondiente aparece 1969, realmente este trabajo de Tkalcu fue publicado en 1970, concretamente el 15 de mayo, a modo de suplemento. El de Rebmann (1970) apareció el 15 de agosto, por lo que los taxones de Tkalcu tienen prioridad.

6 Banaszak y Romasenko (2001) la incluían como sinónima de Chalicodoma lefebvrei Lepeletier, 1841.
Especie paleártica suroccidental. En el territorio mediterráneo occidental está representada por dos subespecies.

Chalicodoma (Allochalicodoma) lefebvrei albida (Pérez, 1897)

Megachile (Chalicodoma) lefebvrei var. albida Pérez, 1897. P.v. Soc. Linn. Bordeaux, 52: 58

Chalicodoma (Chalicodoma) lefebvrei var. albomaculata Friese, 1898. Term. Füz., 21: 201

Subespecie distribuida por la Península Ibérica y Marruecos.

\section{Chalicodoma (Allochalicodoma) lefebvrei lefebvrei Lepe-} letier, 1841

Chalicodoma lefebvrei Lepeletier, 1841. Hist. Nat. Insectes Hyménopt., 2: 332

Chalicodoma luctuosa Dours, 1873. Revue Mag. Zool., 1(3): 299

Megachile (Chalicodoma) muraria var. variabilis Friese, 1920.

Dtsch. Entomol. Z., 1920: 51

Subespecie prácticamente circunmediterránea, citada tanto de Europa meridional (desde Francia hasta Albania) como del norte de África (Argelia y Túnez), así como Córcega, Cerdeña y las islas griegas.

\section{Subgénero Chalicodoma Lepeletier, 1841}

Distribución paleártica suroccidental, a través de Europa central y meridional y la cuenca mediterránea, hasta el Caúcaso.

Chalicodoma (Chalicodoma) albonotata (Radoszkowski, 1886)

?Megachile difficilis Morawitz, 1875. En: Fedtschenko, Izv. Imp. Obsestva. Lûbit. Estestv. Antropol. Etnogr., 19: 106

Megachile albonotata Radoszkowski, 1886. Horae Soc. Entomol. Ross., 20 [1885-1887]: 12

Megachile vachali Benoist, 1934. Bull. Soc. Entomol. France, 39: 109

Chalicodoma cognatum Alfken, 1935. Ent. Rdsch., 52: 162

Chalicodoma strandi Popov, 1936. Festschrift Strand, 1: 591

Megachile (Chalicodoma) pyrenaica var. bytinskii Mavromoustakis, 1949. Eos, 25: 296

Distribuida desde la Península Ibérica hasta el Turkestán. En el área mediterránea occidental se presentan tres subespecies.

\section{Chalicodoma (Chalicodoma) albonotata albonotata} (Radoszkowski, 1886)

Megachile albonotata Radoszkowski, 1886. Horae Soc. Entomol. Ross., 20 [1885-1887]: 12

La subespecie nominal está repartida por el sur de Europa, Asia Menor y Asia Central. 
Chalicodoma (Chalicodoma) albonotata italica Tkalců, 1988

Chalicodoma (Chalicodoma) albonotata italica Tkalců, 1988. Vêst. cs. Spolec. zool., 52: 50

Subespecie exclusiva de Italia (Apeninos y Abruzos).

\section{Chalicodoma (Chalicodoma) albonotata setulosa Pérez, 1895}

Chalicodoma setulosa Pérez, 1895. Espèces Nouv. Mellif. Barbarie: 22

Descrita de "Barbarie", Benoist (1924) restringió su distribución exclusivamente a España.

Chalicodoma (Chalicodoma) apennina (Benoist, 1940)

Megachile muraria var. apennina Benoist, 1940. Ann. Soc. Entomol. France, 109: 43

Exclusiva de Italia (Apeninos y Abruzos).

Chalicodoma (Chalicodoma) baetica Gerstaecker, 1869

Chalicodoma baetica Gerstaecker, 1869. Stett. Entomol. Ztg., 30: 364

Chalicodoma nobilis Dours, 1873. Revue Mag. Zool., 1(3): 298

Distribuida por Europa suroccidental, en la Península Ibérica, Francia y Suiza.

Chalicodoma (Chalicodoma) corsica (Benoist, 1935)

Megachile sicula f. corsica Benoist, 1935. Ann. Soc. Entomol. France, 104: 103

Endémica de Córcega.

Chalicodoma (Chalicodoma) nasidens (Friese, 1898)

Megachile nasidens Friese, 1898. Term. Füz., 21: 201

Megachile geniorum Benoist, 1961. Boll. Mus. Civ. Stor. Nat. Venezia, 14: 49

Especie norteafricana que en la forma nominal se reparte por Argelia; presenta otra población, oriental, que queda fuera del ámbito geográfico estudiado.

Chalicodoma (Chalicodoma) nigrita (Radoszkowski, 1876)

Megachile nigrita Radoszkowski, 1876. Horae Soc. Entomol. Ross., 12(1): 114

Megachile aterrima Pérez, 1895. Espèces Nouv. Mellif. Barbarie: 22

Megachile nigerrima Pérez, 1896. Corr. Espèces Nouv. Mellif. Barbarie: [4]

Distribuida por todo el norte de África, hasta Palestina.

Chalicodoma (Chalicodoma) parietina (Geoffroy, 1785)

Apis parietina Geoffroy, 1785. En: Fourcroy (Ed.), Entomol. Paris., 2: 443
Distribuida por toda Europa (menos el norte) y la cuenca mediterránea, hasta Asia Central.

Chalicodoma (Chalicodoma) parietina nestorea ${ }^{7}$ (Brullé, 1832)

Megachile nestorea Brullé, 1832. En: Bory de Saint Vincent (Ed.), Expéd. Sci. Morée (Insectes), 3(2): 337

Megachile affinis Brullé, 1832. En: Bory de Saint Vincent (Ed.), Expéd. Sci. Morée (Insectes), 3(2): 338

Distribuida desde Italia, incluida Sicilia, hasta Asia Menor y el Cáucaso.

\section{Chalicodoma (Chalicodoma) parietina parietina} (Geoffroy, 1785)

?Apis musciformis Scopoli, 1770. Annus Hist. Nat., 4: 12

?Apis caementaria Meinecke, 1784. Naturforscher, 20: 208

Apis parietina Geoffroy, 1785. En: Fourcroy (Ed.), Entomol. Paris., 2: 443

Apis muraria Geoffroy, 1785. En: Fourcroy (Ed.), Entomol. Paris., 2: 447 (non Retzius, 1783)

?Apis picea Christ, 1791. Nat.gesch. Classif. Nomencl. Insekten: 176

Apis varians Rossi, 1792. Mant. Ins., 1: 142

Megachile aegyptica Lepeletier, 1841. Hist. Nat. Insectes Hyménopt., 2: 331

Chalicodoma leucopogonata Dours, 1873. Revue Mag. Zool., 1(3): 300

Megachile savignyi Radoszkowski, 1874. Bull. Soc. Imp. Nat. Moscou, 48 (1): 150

Chalicodoma valesina Alfken, 1931. Mitt. D. Ent. Ges., 2: 18

Chalicodoma muraria, auctt. (non Retzius, 1783)

Distribuida por Europa central y suroccidental y el norte de África, hasta el límite sahariano.

Chalicodoma (Chalicodoma) pyrenaica (Lepeletier, 1841)

Megachile pyrenaica Lepeletier, 1841. Hist. Nat. Insectes Hyménopt., 2: 336

Ampliamente repartida por Europa (menos el extremo septentrional), el norte de África y, por el este, hasta Kazajistán y el Pamir. En el área mediterránea occidental existen dos subespecies.

Chalicodoma (Chalicodoma) pyrenaica nigricans (Benoist, 1935)

Megachile pyrenaica f. nigricans Benoist, 1935. Ann. Soc. Entomol. France, 104: 102

Subespecie endémica de Italia.

7 Para Tkalců (1970), tanto Chalicodoma nestorea (Brullé, 1832) como Chalicodoma affinis (Brullé, 1832) son sinónimos de Chalicodoma parietina. El estatus actualmente reconocido para ambos taxones, aquí reflejado, lo estableció pocos años después (Tkalců, 1974). 
Chalicodoma (Chalicodoma) pyrenaica pyrenaica (Lepeletier, 1841)

Megachile pyrenaica Lepeletier, 1841. Hist. Nat. Insectes Hyménopt., 2: 336

Megachile rufitarsis Giraud, 1863. Verh. zool.-bot. Ges. Wien., 13: 35 (non Lepeletier, 1841)

Chalicodoma pyrrhopeza Gerstaecker, 1869. Stett. Entomol. Ztg., 30: 366

Subespecie distribuida por Europa central y meridional, y Argelia en el norte de África.

Chalicodoma (Chalicodoma) rufescens Pérez, 1879

Chalicodoma rufescens Pérez, 1879. Act. Soc. Linn. Bordeaux, 33: 219

Chalicodoma pyrenaica var. rufescens, auctt.

Especie exclusiva de Francia y España.

Chalicodoma (Chalicodoma) sicula (Rossi, 1792)

Apis sicula Rossi, 1792. Mant. Ins., 1: 139

Especie circunmediterránea, con varias subespecies en el territorio considerado. Según Liongo li Enkulu (1988), existe en Japón, cita muy extraña que seguramente es errónea, ya que ese autor no aporta datos de captura ni referencia bibliográfica alguna.

Se trata de una especie cuya gran variabilidad ha originado la descripción de un alto número de subespecies correspondientes a poblaciones que en algunos casos se solapan geográficamente. Sin menoscabo de un estudio posterior de material y tipos que ilustren y aclaren estas discordancias, se listan aquí las subespecies encontradas de Chalicodoma sicula en el territorio considerado en este trabajo.

Chalicodoma (Chalicodoma) sicula balearica Tkalců, 1977

Chalicodoma (Chalicodoma) sicula balearica Tkalců, 1977. Vêst. cs. Spolec. zool., 41(3): 236

Van der Zanden (1998; p. 530) reconoce Chalicodoma sicula balearica, subespecie que estaría presente en las Islas Canarias, el norte de África (Marruecos, Argelia y Libia), España, las Islas Baleares y Cerdeña. Otros autores, como Hohmann et al. (1993), también la habían reconocido anteriormente. Curiosamente, en el catálogo de Rasmont et al. (1995) no se trata la subespecie, ni aparece listada entre las sinonimias. Por otro lado, Tkalců (1977), cuando creó esta subespecie, la diferenció sencillamente de la nominal (a la que asignaba la distribución general de la especie) y no aludió a ninguna otra.

\section{Chalicodoma (Chalicodoma) sicula hiendlmayri (Friese,} 1896)

Megachile hiendlmayri Friese, 1896. Term. Füz., 19: 281
Endemismo ibérico, tanto de España como de Portugal.

Chalicodoma (Chalicodoma) sicula perezi (Lichtenstein, 1879)

Megachile sicula var. perezi Lichtenstein, 1879. Ann. Soc. Entomol. France, 9: 166

Chalicodoma pereziana Dalla Torre, 1896. Cat. Hym., 10: 444

Subespecie distribuida por Francia, Córcega y Pirineos en España.

Chalicodoma (Chalicodoma) sicula sicula (Rossi, 1792)

Apis sicula Rossi, 1792. Mant. Ins., 1: 139

Subespecie distribuida en Italia, incluyendo la isla de Sicilia.

\section{Subgénero Euchalicodoma Tkalců, $1970^{8}$}

Chalicodoma (Euchalicodoma) Tkalců, 1970. Acta Mus. Morav., 54 (Suppl.) [1969]: 358

ESPECIE TIPO: Megachile asiatica Morawitz, 1875, por designación original

Megachile (Allomegachile) Rebmann, 1970. Nachr. Bayer. Entomol., 19(3): 42

ESPECIE TIPO: Megachile asiatica Morawitz, 1875, por designación original

Subgénero mediterráneo occidental, que se extiende hasta la región turánica.

\section{Chalicodoma (Euchalicodoma) montenegrensis (Dours,} 1873)

Megachile montenegrensis Dours, 1873. Revue Mag. Zool., 1(3): 304

Megachile syraensis Radoszkowski, 1874. Bull. Soc. Imp. Nat. Moscou, 48(1): 146

?Megachile asiatica Morawitz, 1875. En: Fedtschenko, Izv. Imp. Obsestva. Lûbit. Estestv. Antropol. Etnogr.,19: 105

Chalicodoma ponticum Alfken, 1933. Dtsch. Entomol. Z., 1933: 70

Especie distribuida desde el área mediterránea central hasta el Cáucaso, pero, en el norte de África, sólo conocida en Argelia.

Subgénero Katamegachile Rebmann, 1970

Chalicodoma (Parachalicodoma) Tkalců, 1970. Acta Mus. Morav., 54 (Suppl.) [1969]: 363 (non Pasteels, 1966)

ESPECIE TIPO: Megachile rufitarsis Lepeletier, 1841, por designación original

Megachile (Katamegachile) Rebmann, 1970. Nachr. Bayer. Entomol., 19(3): 43

\footnotetext{
8 Ver nota anterior incluida en Allochalicodoma Tkalců, 1970.
} 
ESPECIE TIPO: Megachile manicata Giraud, 1861, por designación original

Chalicodoma (Xenochalicodoma) Tkalců, 1971. Bull. Soc. Entomol. Mulhouse: 34 (nomen novum para Parachalicodoma Tkalců, 1970)

Katamegachile presenta una distribución circunmediterránea que llega hasta Austria y Hungría en Europa central.

Chalicodoma (Katamegachile) geneana Gribodo, 1894

Chalicodoma geneana Gribodo, 1894. Boll. Soc. entomol. Ital., 26: 96

Distribuida por el norte de África, en Marruecos, Argelia y Túnez. En el área mediterránea occidental presenta dos subespecies.

\section{Chalicodoma (Katamegachile) geneana geneana Gribodo,} 1894

Chalicodoma geneana Gribodo, 1894. Boll. Soc. entomol. Ital., 26: 96

Subespecie endémica de Argelia.

Chalicodoma (Katamegachile) geneana rupestris (Friese, 1899)

Megachile geneana var. rupestris Friese, 1899. Bienen Eur., 5: 197

Subespecie conocida de Marruecos y Argelia.

Chalicodoma (Katamegachile) manicata (Giraud, 1861)

Megachile manicata Giraud, 1861. Verh. zool.-bot. Ges. Wien., 11: 463

?Megachile clavicrus Dours, 1873. Revue Mag. Zool., 1(3): 302

Megachile leucopus Friese, 1898. Term. Füz., 21: 202

Chalicodoma manicata var. fumata Friese, 1913. Arch. Naturg., 78A (12) [1912]: 89

Megachile manicata var. hammanensis Mavromoustakis, 1956. Ann. Mag. Nat. Hist., (123) 9: 862

Especie mediterránea, repartida desde Argelia hasta Palestina y Líbano, incluidos Dalmacia, Grecia (tanto continental como insular), Siria, Tirol y Hungría. Se han descrito una serie de subespecies que, por el momento, no parecen estar bien establecidas.

Chalicodoma (Katamegachile) rufitarsis (Lepeletier, 1841)

Megachile rufitarsis Lepeletier, 1841. Hist. Nat. Insectes Hyménopt., 2: 310

Especie distribuida por el norte de África hasta Túnez, Baleares y la costa dálmata.

Chalicodoma (Katamegachile) rufitarsis maurusia (Vachal, 1910)

Megachile (Chalicodoma) maurusia Vachal, 1910. Bol. R.. Soc. esp. Hist. Nat, 10: 177
Chalicodoma muraria var. tingitana Friese, 1913. Arch. Naturg., 78A (12) [1912]: 88

Subespecie propia de Marruecos.

Chalicodoma (Katamegachile) rufitarsis rufitarsis (Lepeletier, 1841)

Megachile rufitarsis Lepeletier, 1841. Hist. Nat. Insectes Hyménopt., 2: 310

La subespecie nominal tiene una distribución conocida que coincide con la de la especie.

Subgénero Parachalicodoma Pasteels, 1966

Chalicodoma (Parachalicodoma) Pasteels, 1966. Bull. Ann. Soc. R. Belg. Entomol., 102: 13

ESPECIE TIPO: Megachile incana Friese, 1898, por designación original

Subgénero distribuido por Argelia y Egipto.

Chalicodoma (Parachalicodoma) incana (Friese, 1898)

Megachile incana Friese, 1898. Term. Füz., 21: 201

Megachile arlei Benoist, 1943. Bull. Soc. Entomol. France, 48: 88

Especie repartida por Argelia y Egipto.

\section{Subgénero Pseudomegachile Friese, 1898}

Megachile (Pseudomegachile) Friese, 1898. Term. Füz., 21: 198 ESPECIE TIPO: Megachile ericetorum Lepeletier, 1841, por designación de Alfken, 1933

Megachile (Pseudomegalochila) Schulz, 1906. Spolia Hymenopterol.: 71, enmienda injustificada

Megachile (Archimegachile) Alfken, 1933. Konowia, 12: 56

ESPECIE TIPO: Megachile flavipes Spinola, 1839, por designación original

Chalicodoma (Neglectella) Pasteels, 1965. Ann. Mus. R. Afriq. Centr., Sci. Zool., 137: 431

ESPECIE TIPO: Megachile armatipes Friese, 1909, por designación original

Chalicodoma (Dinavis) Pasteels, 1965. Ann. Mus. R. Afriq. Centr., Sci. Zool., 137: 549

ESPECIE TIPO: Megachile muansae Friese, 1911 por designación original

Megachile (Xenomegachile) Rebmann, 1970. Nachr. Bayer. Entomol., 19(3): 44

ESPECIE TIPO: Megachile albocincta Radoskowski, 1874, por designación original

Subgénero con una amplia distribución que excede la región paleártica, pues se extiende hasta Suráfrica y la India y el sureste de Asia, además de grandes islas como Madagascar y Reunión; ha sido introducido en las Antillas y hay una especie citada en Florida (EE.UU.). Incluye unas 50 especies del África subsahariana y más de 30 en el resto de su territorio (Pasteels, 1965; Michener, 2000). 
Chalicodoma (Pseudomegachile) cinnamomea Alfken, 1926

Megachile cinnamomea Alfken, 1926. Senckenbergiana, 8: 125

Distribuida por África noroccidental, en Mauritania, Marruecos, Argelia, y Egipto.

Chalicodoma (Pseudomegachile) ericetorum (Lepeletier, 1841)

?Apis barbata Schrank, 1802. Faun. Boica, 2(2): 380

Megachile ericetorum Lepeletier, 1841. Hist. Nat. Insectes Hyménopt., 2: 341

Megachile cristata Dufour, 1841. Mém. Acad. Sci. Inst. Fr., 7: 420

Megachile fasciata Smith, 1844. Zoologist, 2: 694

Megachile rufitarsis Smith, 1844. Zoologist, 2: 695 (non Lepeletier, 1841)

Megachile pyrina Nylander, 1852. Not. Sällsk. Fauna Flora Fenn. Förh., 2 (Revis.): 275 (non Lepeletier, 1841)

Megachile senex Smith, 1853. Cat. Hymenopt. Insects Collect. Br. Mus., 1: 160

Megachile tsingtauensis Strand, 1915. Ent. Mitt., 4: 75

Especie paleártica occidental, con una serie de subespecies geográficas de las que, en el territorio estudiado, aparecen las tres siguientes.

\section{Chalicodoma (Pseudomegachile) ericetorum euroa Tkalců, 1988}

Chalicodoma (Pseudomegachile) ericetorum euroa Tkalců, 1988. Vêst. cs. Spolec. zool., 52: 49

Subespecie endémica de Argelia.

\section{Chalicodoma (Pseudomegachile) ericetorum melaleuca} van der Zanden, 1989

Chalicodoma (Pseudomegachile) ericetorum melaleuca van der Zanden, 1989. Entomol. Abh. Mus. Tierkd. Dresden, 53(6): 72

Subespecie mediterránea occidental, distribuida por España, Córcega y Marruecos.

\section{Chalicodoma (Pseudomegachile) ericetorum oraniensis} van der Zanden, 1989

Chalicodoma (Pseudomegachile) ericetorum oraniensis van der Zanden, 1989. Entomol. Abh. Mus. Tierkd. Dresden, 53(6): 72

Subespecie del noroeste de África, repartida por Marruecos y Argelia.

Chalicodoma (Pseudomegachile) flavipes Spinola, 1839

Megachile flavipes Spinola, 1839. Ann. Soc. Entomol. France, 7(4) [1838]: 527

Megachile tecta Radoszkowski, 1888. Horae Soc. Entomol. Ross., 22: 339
Distribuida por el norte de África, desde Argelia, hasta Asia Central y el Turkestán (incluida la isla de Chipre).

Chalicodoma (Pseudomegachile) hungarica Mocsáry, 1877

Chalicodoma hungarica Mocsáry, 1877. Petit. Nouv. Ent., 2: 109

Chalicodoma libanensis Pérez, 1910. Bull. Soc. Amis Sci. Nat. Rouen, 46: 39

Chalicodoma fallax Alfken, 1932. Bull. Soc. Roy. Ent. Egypte: 223

Chalicodoma podolica Noskiewicz, 1936. Polski. Pismo. Entomol., 13(1/4) [1934]: 152, 167

Chalicodoma lefebvrei luridiscopa Alfken, 1938. Dtsch. Entomol. Z., 1938: 424

Especie distribuida por Europa suroriental, Palestina, Israel, Líbano, Asia Menor y, en el norte de África, en Argelia.

Chalicodoma (Pseudomegachile) luteipennis (Friese, 1898)

Megachile luteipennis Friese, 1898. Term. Füz., 21: 202

Especie endémica de Argelia.

Chalicodoma (Pseudomegachile) marginata (Smith, 1853)

Megachile marginata Smith, 1853. Cat. Hymenopt. Insects Collect. Br. Mus., 1: 151

?Megachile pugillatoria Costa, 1863. Atti. Accad. Sc. fis. mat. Napoli, 1(2): 43

Megachile derasa Gerstaecker, 1869. Stett. Entomol. Ztg., 30: 361

Stelis megachiloides Alfken, 1942. Veröff. dt. Kolon. u.Übersee-Mus. Bremen, 3(3): 215

Especie distribuida por el área mediterránea central y oriental, desde Italia (Calabria), que llega al Cáucaso y, en el norte de África, se ha citado de Egipto.

Chalicodoma (Pseudomegachile) nigripes (Spinola, 1839)

Megachile nigripes Spinola, 1839. Ann. Soc. Entomol. France, 7(4) [1838]: 529

Megachile nilotica Pérez, 1897. P.-v. Soc. Linn. Bordeaux, 52: 60

Megachile mucorea Friese, 1898. Term. Füz., 21: 200

Distribuida por el norte de África, en Argelia y Egipto.

\section{Incertae sedis}

Megachile lucidifrons Ferton, 1905

Megachile lucidifrons Ferton, 1905. Annls. Soc. ent. Fr. 74: 57

Se trata de una especie descrita con un solo ejemplar de Córcega (Bonifacio) y de la que no se han vuelto a aportar datos. Sólo se conoce una hembra, que no se ha revisado, por lo que no se puede descartar su validez. Rasmont et al. (1995) la adscribían al subgénero Chalicodoma (Allochalicodoma), y bien podría ser una forma de 
Chalicodoma (Allochalicodoma) lefebvrei (Hartmann, com. pers.).

Megachile ghilianii Spinola, 1843

Megachile ghilianii. Spinola, 1843. Ann. Soc. Entomol. France, (2)1 (2): 142

De esta especie sólo se sabe que fue descrita con una hembra de "España", cita que recoge Ceballos en su catálogo de 1956. Nada se ha aportado desde aquel momento, y su posible inclusión en el subgénero nominal de Chalicodoma se basaría en la indicación que realizó el mismo autor en su descripción, por su proximidad con "Megachile pyrenaica".

\section{Chalicodoma sefrensis (Benoist, 1943)}

Megachile (Chalicodoma) sefrensis Benoist, 1943. Bull. Soc. Entomol. France, 48: 88

Especie descrita con material de Sefra (Argelia) y sobre la que no se han vuelto a aportar datos desde su descripción.

\section{Megachile breviceps (Friese, 1898)}

Megachile breviceps Friese, 1898. Term. Füz., 21: 200

Esta especie fue descrita con un solo ejemplar, una hembra de "Hispania" capturada por Schmiedecknecht. El autor (Friese, 1898) indicaba que se "asemeja a Megachile flaviceps", lo que haría que pudiera ser incluida en el subgénero Chalicodoma (Pseudomegachile). Pasteels (1966) planteaba, aunque con muchas dudas, que podría tratarse de un miembro del subgénero Chalicodoma (Callomegachile) Michener, 1962. Igualmente, Michener (2000) la incluyó en Megachile (Callomegachile), pero apuntando que, en ese caso, podría ser un ejemplar mal etiquetado, pues Callomegachile es un subgénero tropical.

Megachile algira Radoszkowski, 1874

Megachile algira Radoszkowski, 1874. Bull. Soc. Imp. Nat. Moscou, 48(1): 144 (non Friese, 1898)

Descrita de Argelia, Pérez (1897) indica su pertenencia a Chalicodoma, con semejanzas con Chalicodoma muraria. Alfken, en un artículo sobre la fauna de Argelia (1914), no la menciona, ni aportando datos ni reflejando las citas precedentes. Por otra parte, Baker (1993) opina que la especie es sinónima de Chalicodoma (Pseudomegachile) florale oppositum ( $\mathrm{Sm}$.), en cuyo caso la localidad típica sería "Java", y no "Algerie". Sin embargo, ese autor no aclara si la sinonimia la hace previo el estudio del material típico, lo que no aclara nada la situación.

\section{Megachile transitoria Benoist, 1934}

Megachile transitoria Benoist, 1934. Bull. Soc. Entomol. France, 39: 108
Especie descrita a partir de una hembra procedente de Azrou (Marruecos). No se han vuelto a aportar datos, ni morfológicos ni de distribución. El propio autor (Benoist, 1935) la compara con "Megachile muraria, $M$. pyrenaica y M. rufitarsis" (que son especies o sinónimos de especies de Chalicodoma). No se tienen otros datos.

\section{Megachile jeanneli Benoist, 1934}

Megachile jeanneli Benoist, 1934. Bull. Soc. Entomol. France, 39: 109

Descrita con material de Argelia, no ha vuelto a citarse. Benoist (1935) la compara con Megachile nasidens, lo que podría apuntar a su pertenencia al género Chalicodoma.

Género Megachile Latreille, 1802

Megachile Latreille, 1802. Hist. Nat. Fourmis: 413, 433

ESPECIE TIPO: Apis centuncularis Linnaeus, 1758, por designación de Curtis, 1828

Anthophora Fabricius, 1804. Syst. Piez.: 372 (non Latreille, 1803)

ESPECIE TIPO: Apis centuncularis Linnaeus, 1758, por designación de Michener, 1951

Anthemois Robertson, 1903. Trans. Am. Entomol. Soc., 29: 168,172

ESPECIE TIPO: Megachile infragilis Cresson, $1878=$ Apis centuncularis Linnaeus, 1758, por designación original

Cyphopyga Robertson, 1903. Trans. Am. Entomol. Soc., 29: 169,172

ESPECIE TIPO: Megachile montivaga Cresson, 1878, por designación original

Megalochila Schulz, 1906. Spolia Hymenopterol.: 263, enmienda injustificada de Megachile Latreille, 1802

Género distribuido prácticamente por todo el mundo (Michener, 2000).

Subgénero Eutricharaea Thomson, 1872

Megachile (Eutricharaea) Thomson, 1872. Hym. Scand., 2: 228

ESPECIE TIPO: Apis argentata Fabricius, 1793, por monotipia

Megachile (Paramegachile) Friese, 1898. Term. Füz., 21: 198 (partim)

ESPECIE TIPO: Apis argentata Fabricius, 1793, por designación de Mitchell, 1934

Megachile (Paramegalochila) Schulz, 1906. Spolia Hymenopterol:: 71, enmienda injustificada

Androgynella Cockerell, 1911. Ann. Mag. Nat. Hist., 8(7): 313 ESPECIE TIPO: Megachile detersa Cockerell, 1910, por designación original

Perezia Ferton, 1914. Ann. Soc. Entomol. France, 83: 233 (non Léger \& Dubosq, 1909)

ESPECIE TIPO: Perezia maura Ferton, 1914 = Megachile leachella Curtis, 1828, por monotipia

Fertonella Cockerell, 1920. Ann. Durban Museum, 2(5): 257 (nomen novum para Perezia Ferton, 1914) 
ESPECIE TIPO: Perezia maura Ferton, $1914=$ Megachile leachella Curtis, 1828, por designación original

Megachile (Eurymella) Pasteels, 1965. Ann. Mus. R. Afriq. Centr., Sci. Zool., 137: 64

ESPECIE TIPO: Megachile eurymera Smith, 1854, por designación original

Megachile (Digitella) Pasteels, 1965. Ann. Mus. R. Afriq. Centr., Sci. Zool., 137: 191

ESPECIE TIPO: Megachile digiticauda Cockerell, 1937, por designación original

Megachile (Melaneutricharaea) Tkalců, 1993. Veröff. Übersee-Mus. Bremen (Naturwiss.), 12: 803

ESPECIE TIPO: Megachile hohmanni Tkalců, 1993, por designación original

Megachile (Anodonteutricharaea) Tkalců, 1993. Veröff. Übersee-Mus. Bremen (Naturwiss.), 12: 807

ESPECIE TIPO: Megachile larochei Tkalců, 1993 = Megachile lanigera Alfken, 1933, por designación original

Distribución paleártica, africana, oriental y australiana, desde regiones templadas hasta desiertos y zonas tropicales; introducido en América, Antillas y Nueva Zelanda (Michener, 2000).

\section{Megachile (Eutricharaea) albipila Pérez, 1896}

Megachile mucida Pérez, 1895. Espèces Nouv. Mellif. Barbarie: 23 (non Cresson, 1878)

Megachile albipila Pérez, 1896. Corr. Espèces Nouv. Mellif. Barbarie: [X]

Descrita de "Barbarie", se distribuye, por el ámbito mediterráneo, a través de todo el norte de África, Grecia y Palestina.

Megachile (Eutricharaea) albohirta (Brullé, 1839)

Osmia (Megachile) albo-hirta Brullé, 1839. En: Webb \& Berthelot, Hist. nat. Iles Canar., 2: 86

Megachile variscopa Pérez, 1895. Espèces Nouv. Mellif. Barbarie: 24

Megachile concinna Smith, 1879. Descr. New Spec. Hymen.: 79

Distribuida desde las Canarias, por todo el norte de África, y desde España hasta Italia (Córcega incluida).

\section{Megachile (Eutricharaea) atratula Rebmann, 1968}

Megachile (Eutricharaea) atratula Rebmann, 1968. Dtsch. Entomol. Z., (N.F.), 15: 40

Especie mediterránea occidental (Italia, Sicilia, Francia, Iberia, Marruecos), presente también en las Azores.

Megachile (Eutricharaea) inexspectata Rebmann, 1968

Megachile (Eutricharaea) inexspectata Rebmann, 1968. Dtsch. Entomol. Z., (N.F.), 15: 43

Distribución en África noroccidental (Argelia) y Asia Menor.
Megachile (Eutricharaea) lanigera Alfken, 1933

Megachile lanigera Alfken, 1933. Mitt. D. Ent. Ges., 4: 133

Megachile (Anodonteutricharaea) larochei Tkalců, 1993. Veröff. Übersee-Mus. Bremen (Naturwiss.), 12: 807

Distribuida por Marruecos y las Islas Canarias.

Megachile (Eutricharaea) leachella Curtis, 1828

?Apis albiventris Panzer, 1798. Faunae Insector. Ger., 56: $\mathrm{n}^{\circ}$ 19 (non Christ, 1791)

Megachile leachella Curtis, 1828. British Entomol., 5: 219

Megachile saussurei Radoszkowski, 1874. Bull. Soc. Imp. Nat. Moscou, 48(1): 142

Megachile multispinosa Morawitz, 1875. En: Fedtschenko, Izv. Imp. Obsestva. Lûbit. Estestv. Antropol. Etnogr., 19: 118

Megachile argentata var. fossoria Ferton, 1909. Ann. Soc. Entomol. France, 77 [1908]: 550

Perezia maura Ferton, 1914. Ann. Soc. Entomol. France, 83: 233 (non Cresson, 1865)

Megachile argentata, auctt. (non Fabricius, 1793)

Especie paleártica occidental.

Véase Hurd (1967), entre otros, en relación con la designación como "Megachile argentata" que gran número de autores daban, incluso no hace mucho tiempo, a Megachile leachella.

\section{Megachile (Eutricharaea) pilidens Alfken, 1924}

Megachile pilidens Alfken, 1924. Rozpr. Wiad. Muz. Dziedusz., 9 [1923]: 88

Megachile argyrea Cockerell, 1931. Ann. Mag. Nat. Hist., 10(7): 275

Distribuida por casi toda Europa, excepto el extremo septentrional, y el norte de África.

Megachile (Eutricharaea) schmiedeknechti Costa, 1884

Megachile schmiedeknechti Costa, 1884. Rendic. Accad. Sc. fis. mat. Napoli, 23: 169

Megachile xanthopyga Pérez, 1895. Espèces Nouv. Mellif. Barbarie: 25

Especie mediterránea occidental, repartida por Francia, Córcega, Cerdeña, Italia, Argelia y Marruecos.

Megachile (Eutricharaea) sexmaculata Alfken, 1942

Megachile (Eutricharaea) sexmaculata Alfken, 1942. Veröff. dt. Kolon. u. Übersee-Mus. Bremen, 3(3): 210

Especie endémica de Argelia.

Megachile (Eutricharaea) striatella Rebmann, 1968

Megachile (Eutricharaea) striatella Rebmann, 1968. Dtsch Entomol. Z., (N.F.), 15: 41

Distribución prácticamente circunmediterránea; no citada, de momento, de Francia e Italia, pero llega por el este hasta Afganistán y, en el sur, se conoce aisladamente en Suráfrica (Transvaal). 


\section{Subgénero Megachile Latreille, 1802}

Subgénero holártico de zonas frías o subártico (hasta Alaska), aunque también existe en EE.UU. (Florida y California) y Méjico. Sólo una especie, Megachile centuncularis (Linnaeus, 1758), es holártica.

\section{Megachile (Megachile) alpicola Alfken, 1924}

Megachile alpicola Alfken, 1924. Dtsch. Entomol. Z.,1924: 357

Megachile rubtzovi Cockerell, 1928. Ann. Mag. Nat. Hist., 10(1): 357

Especie europea, principalmente de la franja media, que llega por el sur hasta los Pirineos, los Alpes y el Monte Olimpo, en Grecia; su distribución se extiende por el este hasta Siberia y el Cáucaso.

Megachile (Megachile) centuncularis (Linnaeus, 1758) Apis centuncularis Linnaeus, 1758. Syst. Nat., ed. 10, 1: 575

Especie holártica que en el territorio estudiado presenta dos subespecies.

\section{Megachile (Megachile) centuncularis centuncularis} (Linnaeus, 1758)

Apis centuncularis Linnaeus, 1758. Syst. Nat., ed. 10, 1: 575 Megachile parvula Lepeletier, 1841. Hist. Nat. Insectes Hyménopt., 2: 340

Megachile infragilis Cresson, 1878. Trans. Amer. Ent. Soc., 7: 127

Megachile leoni Titus, 1906. Proc. Ent. Soc. Wash., 7 [1905]: 150

Subespecie europea que por el sur llega hasta España y falta en el extremo más septentrional.

\section{Megachile (Megachile) centuncularis theryi Cockerell,} 1931

Megachile centuncularis theryi Cockerell, 1931. Ann. Mag. Nat. Hist., 10(7): 274

Subespecie endémica de Marruecos.

Megachile (Megachile) genalis Morawitz, 1880

Megachile genalis Morawitz, 1880. Bull. Acad. Imp. Sci. St. Petersbourg, 26: 380

Megachile villosa Henschel, 1888. Ent. Nachr., 14: 321 (non Fabricius, 1775; nec Schenck, 1853)

Megachile henscheli Dalla Torre, 1896. Cat. Hym., 10: 433 (nomen novum para Megachile villosa Henschel, 1888)

Especie distribuida por Europa central y meridional, hasta Asia Central. En el territorio estudiado existe una única subespecie; la forma nominal existe sólo en Suiza.

Megachile (Megachile) genalis buyssoni Pérez, 1890

Megachile buyssoni Pérez, 1890. Act. Soc. Linn. Bordeaux, 44: 190
Subespecie de Europa suroccidental.

Megachile (Megachile) lapponica Thomson, 1872

Megachile lapponica Thomson, 1872. Hym. Scand., 2: 227

Megachile melanopyga amaguella Cockerell, 1924. Ann.. Mag. Nat. Hist., 9(13): 602

Especie distribuida en Eurasia sobre todo por latitudes altas, pero existe en Francia y, en altitud, en Grecia.

\section{Megachile (Megachile) ligniseca (Kirby, 1802)}

Apis ligniseca Kirby, 1802. Monogr. Apum Angl., 2: 243

?Apis catula Gravenhorst, 1807. Vergl. Übers. Zool. Syst.: 284

?Apis nigella Gravenhorst, 1807. Vergl. Übers. Zool. Syst.: 285

Especie europea cuya distribución llega hasta el Cáucaso. Presenta una serie de subespecies, de las que sólo la nominal vive en el territorio mediterráneo occidental, y está distribuida por Europa occidental, desde Francia hasta Rumanía, y el Cáucaso.

\section{Megachile (Megachile) melanopyga Costa, 1863}

Megachile melanopyga Costa, 1863. Atti. Accad. Sc. fis. mat. Napoli, 1(2): 45

Megachile vestita Giraud, 1863. Verh. zool.-bot. Ges. Wien., 13: 35 (non Smith, 1853)

Megachile hymenaea Gerstaecker, 1869. Stett. Entomol. Ztg., 30: 356

Distribuida por toda Europa excepto el norte, hasta Siberia y el Cáucaso; la forma nominal aparece en Europa occidental; una referencia de Japón y Corea (Banaszak \& Romasenko, 2001) quizá debe ser revisada

Megachile (Megachile) octosignata Nylander, 1852

Megachile octosignata Nylander, 1852. Not. Sällsk. Fauna Flora Fenn. Förh., 2 (Revis.): 274 Cáucaso.

Distribuida prácticamente por toda Europa, y el Megachile (Megachile) pilicrus Morawitz, 1879

Megachile pilicrus Morawitz, 1879. Horae Soc. Entomol. Ross., 14 [1878]: 52

Megachile vicina Mocsáry, 1879. Term. Füz., 3: 8

Distribuida por toda Europa, excepto el extremo norte, y el Cáucaso.

\section{Megachile (Megachile) pyrenaea Pérez, 1890}

Megachile pyrenaea Pérez, 1890. Act. Soc. Linn. Bordeaux, 44: 192

Distribuida por Europa hasta Turquía y el Cáucaso, desde Francia hasta Finlandia y Suecia, pero sólo con carácter orófilo en España y Grecia. 
Megachile (Megachile) versicolor Smith, 1844

Megachile versicolor Smith, 1844. Zoologist, 2: 697

Distribuida por Europa meridional y central, desde España y Francia hasta Rumanía, por el norte hasta Irlanda y por el este hasta Siberia y Turquía.

Subgénero Neoeutricharaea Rebmann, 1967

Megachile (Neoeutricharaea) Rebmann, 1967. Ent. Zeit., 77: 36 ESPECIE TIPO: Apis rotundata Fabricius, 1787, por designación original

Megachile (Neoeutricharaea) apicalis Spinola, 1808

Megachile apicalis Spinola, 1808. Insect. Ligur., 2: 259

Megachile mixta Costa, 1863. Atti. Accad. Sc. fis. mat. Napoli, 1(2): 44

Megachile dimidiativentris Dours, 1873. Revue Mag. Zool., 1(3): 300

Megachile massiliensis Pérez, 1902. P.-v. Soc. Linn. Bordeaux, 57: 121

Megachile virginiana Mitchell, 1937. Trans. Am. Ent. Soc., 63: 417

Distribuida por el centro y sur de Europa, incluyendo Chipre y Rodas, y norte de África, hasta Asia Central; introducida en Canadá.

Megachile (Neoeutricharaea) burdigalensis Benoist, 1940

Megachile burdigalensis Benoist, 1940. Ann. Soc. Entomol. France, 109: 78

Megachile dorsalis Pérez, 1879. Act. Soc. Linn. Bordeaux, 33: 223, ơ (non 우)

Especie distribuida por Francia, Eslovenia y, posiblemente, Kazajistán (según Gogala, 1998).

Sólo se conocía la serie típica (dos machos), de Burdeos (Francia), hasta que Gogala (1998) designó el lectotipo y describió la hembra. Rasmont et al. (1995) la situaban en el subgénero Eutricharaea, pero es, claramente, un representante de Neoeutricharaea, muy próximo a Megachile flabellipes Pérez, 1895.

Megachile (Neoeutricharaea) crassula Pérez, 1896

Megachile compacta Pérez, 1895. Espèces Nouv. Mellif. Barbarie: 24 (non Smith, 1879)

Megachile crassula Pérez, 1896. Corr. Espèces Nouv. Mellif. Barbarie: [X]

Megachile beaumonti Benoist, 1950. Bull. Soc. Scienc. Nat. Maroc, 30: 189

Descrita de "Barbarie", se distribuye por África noroccidental en Marruecos y Argelia.

\section{Megachile (Neoeutricharaea) deceptoria Pérez, 1890}

Megachile deceptoria Pérez, 1890. Act. Soc. Linn. Bordeaux, 44: 192
Megachile mogadorensis Benoist, 1934. Bull. Soc. Entomol. France, 39: 109

Distribuida por Europa meridional, hasta Kazajistán, y el norte de África, en Marruecos y Argelia.

Megachile (Neoeutricharaea) dorsalis Pérez, 1879

Megachile dorsalis Pérez, 1879. Act. Soc. Linn. Bordeaux, 33: 223

Distribuida por Europa meridional: desde Portugal hasta Hungría, incluyendo Austria.

Megachile (Neoeutricharaea) fertoni Pérez, 1895

Megachile fertoni Pérez, 1895. Espèces Nouv. Mellif. Barbarie: 23

Descrita de "Barbarie", se distribuye por el área mediterránea occidental, Islas Baleares incluidas.

Megachile (Neoeutricharaea) flabellipes Pérez, 1895

?Megachile albopicta Smith, 1853. Cat. Hymenopt. Insects Collect. Br. Mus., 1: 154

Megachile flabellipes Pérez, 1895. Espèces Nouv. Mellif. Barbarie: 23

Especie mediterránea occidental, aunque no citada en todos los países, distribuida hasta Eslovaquia y Austria.

Megachile (Neoeutricharaea) gothalauniensis Pérez, 1902

Megachile gothalauniensis Pérez, 1902. P.-v. Soc. Linn. Bordeaux, 57: 120

Especie descrita de España, sobre la que no se han vuelto a aportar nuevos datos de distribución.

Megachile (Neoeutricharaea) leucomalla Gerstaecker, 1869

Megachile leucomalla Gerstaecker, 1869. Stett. Entomol. Ztg., 30: 360

Megachile excellens Morawitz, 1872. Horae Soc. Entomol. Ross., 9: 53

Megachile laevifrons Morawitz, 1879. Horae Soc. Entomol. Ross., 14 [1878]: 53

Megachile sedilloti Pérez, 1895. Espèces Nouv. Mellif. Barbarie: 24

Megachile leucomalla var. panzeri Friese, 1899. Bienen Eur., 5: 48

Distribuida por Europa meridional, hasta el sur de Rusia, Turquía y Jordania y, en el norte de África, en Argelia.

Tkalců (1967) y van der Zanden (1983) la incluían en el subgénero Eutricharea. 
Megachile (Neoeutricharaea) melanogaster Eversmann, 1852

Megachile melanogaster Eversmann, 1852. Bull. Soc. Imp. Nat. Moscou, 25: 73.

Especie repartida por Francia, Suiza y Rusia, en Europa, y Argelia, en el norte de África; una cita dudosa de España (Pérez-Íñigo, 1984) ha sido confirmada recientemente por Müller \& Bansac (2004).

Megachile (Neoeutricharaea) opacifrons Pérez, 1897

Megachile opacifrons Pérez, 1897. P.-v. Soc. Linn. Bordeaux, 52: 64

Especie conocida sólo de Francia.

Megachile (Neoeutricharaea) picicornis Morawitz, 1879

?Megachile marginata Smith, 1853. Cat. Hymenopt. Insects Collect. Br. Mus., 1: 151

?Megachile panzeri Dours, 1873. Mém. Soc. linn. N. France, 3 [1872-1873]: 194

Megachile picicornis Morawitz, 1879. Horae Soc. Entomol. Ross., 14 [1878]: 55

Megachile albocincta Pérez, 1879. Act. Soc. Linn. Bordeaux, 33: 224 (non Radoszkowski, 1874)

Megachile morawitzi Radoszkowski, 1886. Horae Soc. Entomol. Ross., 20 [1885-1887]: 11

Megachile perezi Mocsáry, 1887. Term. Füz., 11: 19 (nomen novum para Megachile albocincta Pérez, 1879)

Megachile provincialis Pérez, 1890. Act. Soc. Linn. Bordeaux, 44: 193 (non Lichtenstein, 1879) (nomen novum para Megachile albocincta Pérez, 1879)

Distribuida en el área mediterránea, desde la Península Ibérica (España y Portugal), Francia y Chipre y África septentrional hasta el Turkestán.

\section{Megachile (Neoeutricharaea) pruinosa Pérez, 1897}

Megachile pruinosa Pérez, 1897. P.-v. Soc. Linn. Bordeaux, 52: 63 (non Friese, 1903)

Especie conocida sólo por su holotipo de Aviñón, Francia (una hembra, el macho es desconocido). Los caracteres diferenciadores aportados por Benoist (1940) son consistentes y la separan bien de las especies próximas, aunque se hace necesario volver a capturarla, sobre todo el macho.

Megachile (Neoeutricharaea) rotundata (Fabricius, 1787)

Apis rotundata Fabricius, 1787. Mant. Ins., 1: 303 (non Panzer, 1798)

Apis pacifica Panzer, 1798. Faunae Insector. Ger., 55: $\mathrm{n}^{\circ} 16$

Megachile imbecilla Gerstaecker, 1869. Stett. Entomol. Ztg., 30: 359

?Megachile pusilla Pérez, 1883. Act. Soc. Linn. Bordeaux, 37: 263

?Megachile nadia Nurse, 1903. Ann. Mag. Nat. Hist., 7(11): 546
Distribuida por casi toda Europa y, en África, en Marruecos; introducida en Norte y Suramérica y Nueva Zelanda.

\section{Subgénero Xanthosarus Robertson, 1903}

Xanthosarus Robertson, 1903. Trans. Am. Entomol. Soc., 29: $168,169,172$

ESPECIE TIPO: Megachile latimanus Say, 1823, por designación original

Megachile (Delomegachile) Vierek, 1916. Connont. Stat. Geol. Nat. Hist. Surv. Bull., 22: 745

ESPECIE TIPO: Megachile vidua Smith, 1853 = Megachile latimanus Say, 1823, por monotipia

Megachile (Phaenosarus) Mitchell, 1934. Trans. Am. Entomol. Soc., 59: 303, 309

EsPECIE TIPO: Megachile fortis Cresson, 1872, por designación original

Megachile (Macromegachile) Noskiewicz, 1948. Polski. Pismo. Entomol., 18: 48

ESPECIE TIPO: Apis lagopoda Linnaeus, 1761, por designación original

Megachile (Addendella) Mitchell, 1980. A Gen. Rev. Megach. Bees West. Hemisph.: 24

EsPECIE TIPO: Megachile addenda Cresson, 1878, por designación original

Cressoniella (Orientocressoniella) Gupta, 1993. Taxon. Stud. Megach. N-W India: 165

ESPECIE TIPO: Megachile relata Smith, 1879, por designación original

Subgénero holártico; en la región neártica existe en Norteamérica y Méjico y, en la paleártica, se distribuye desde España hasta el Reino Unido por el norte y, por el este, hasta Japón (Michener, 2000). Incluye unas 13 especies euroasiáticas.

Megachile (Xanthosarus) analis Nylander, 1852

Megachile apicalis Nylander, 1848. Not. Sällsk. Fauna Flora Fenn. Förh., 1 (Adnot.): 257 (non Spinola, 1808)

Megachile analis Nylander, 1852. Not. Sällsk. Fauna Flora Fenn. Förh., 2 (Revis.): 275

Megachile albicilla Eversmann, 1852. Bull. Soc. Imp. Nat. Moscou, 25: 71

Megachile obscura Eversmann, 1852. Bull. Soc. Imp. Nat. Moscou, 25: 71

Megachile analis var. albida Friese, 1898. Term. Füz., 21: 198 Megachile kuennemannii Alfken, 1897. Ent. Nachr., 23: 161

Megachile kuennemannii var. obscura Alfken, 1897. Ent. Nachr., 23: 162 (non Eversmann, 1852)

Megachile angarensis Cockerell, 1928. Ann. Mag. Nat. Hist., 10(1): 354

Megachile analis var. holsatica Alfken, 1936. Mitt. D. Ent. Ges., 6 [1935]: 59 (nomen novum para Megachile analis var. obscura Alfken, 1897)

Distribuida por toda Europa, incluida España en el sur, hasta Siberia y el Turquestán. Según Ebmer (2001), además de la subespecie nominal, que es la que aparece en el territorio estudiado, existen otras seis subespecies, todas ellas asiáticas. Para Rasmont et al. (1995), a dife- 
rencia de Tkalců (1975), Schwarz et al. (1996) o Ebmer (2001), Megachile (Xanthosarus) analis kuennemannii Alfken, 1897 es una buena subespecie de Suiza, de la que también Ceballos (1956) referenció dos citas ibéricas de Huesca y Cádiz.

\section{Megachile (Xanthosarus) atlantica Benoist, 1934}

Megachile atlantica Benoist, 1934. Bull. Soc. Entomol. France, 39: 108

Distribuida en África noroccidental, por Marruecos y Argelia, y Sicilia.

Megachile (Xanthosarus) circumcincta (Kirby, 1802)

Apis circumcincta Kirby, 1802. Monogr. Apum Angl., 2: 235

Distribuida por Gran Bretaña, Europa septentrional y central, a través del entorno mediterráneo, por Turquía y Sicilia y por Argelia, en el norte de África.

\section{Megachile (Xanthosarus) circumcincta circumcincta} (Kirby, 1802)

?Apis bryorum Schrank, 1781. Enum. Ins. Austr.: 402 (non Fabricius, 1775)

Apis circumcincta Kirby, 1802. Monogr. Apum Angl., 2: 246

Apis willughbiella var. $\gamma$ Kirby, 1802. Monogr. Apum Angl., 2: 235

Megachile circumcincta var. insidiosa Benoist, 1940. Ann.

Soc. Entomol. France, 109: 49

Subespecie distribuida por Europa central y septentrional, excepto el extremo norte, y en el sur, incluida España, sólo en altitud.

Megachile (Xanthosarus) circumcincta etnaensis van der Zanden, 1989

Megachile (Macromegachile) circumcincta etnaensis van der Zanden, 1989. Entomol. Abh. Mus. Tierkd. Dresden, 53(6): 73

Subespecie exclusiva de Sicilia.

\section{Megachile (Xanthosarus) circumcincta numidica Tkalců,} 1988

Megachile (Delomegachile) circumcincta numidica Tkalců, 1988. Vêst. cs. Spolec. zool., 52: 57

Subespecie endémica de Argelia.

Megachile (Xanthosarus) giraudi Gerstaecker, 1869

Megachile giraudi Gerstaecker, 1869. Stett. Entomol. Ztg., 30: 355

Distribuida por Europa meridional, desde España hasta Turquía.

Megachile (Xanthosarus) giraudi giraudi Gerstaecker, 1869

Megachile giraudi Gerstaecker, 1869. Stett. Entomol. Ztg., 30: 355
Subespecie repartida en Europa suroccidental por España, Francia y Suiza.

\section{Megachile (Xanthosarus) giraudi intermixta Gerstaecker, 1869}

Megachile intermixta Gerstaecker, 1869. Stett. Entomol. Ztg., 30: 358

Megachile bicoloriventris Mocsáry, 18789. Term. Füz., 2: 122

Subespecie repartida en Europa suroriental desde Italia continental y Sicilia, hasta Turquía.

Megachile (Xanthosarus) lagopoda (Linnaeus, 1761)

Apis lagopoda Linnaeus, 1761. Fauna Suecica, ed. 2: 422

Distribución prácticamente paleártica occidental, a excepción de los extremos norte y este.

Megachile (Xanthosarus) lagopoda lagopoda (Linnaeus, 1761)

Apis lagopoda Linnaeus, 1761. Fauna Suecica, ed. 2: 422

Apis lagopus Gmelin, 1790. Linné Syst. Nat., ed.13, 1(5): 2776 (nomen novum para Apis lagopoda Linnaeus, 1761)

?Anthophora rufiventris Fabricius, 1804. Syst. Piezator: 378

Megachile pyrina Lepeletier, 1841. Hist. Nat. Insectes Hyménopt., 2: 334 (non Nylander, 1852)

Megachile lagopoda var. nigricans Alfken, 1914. Mém. Soc. ent. Belg., 22: 207

Megachile baleina Cockerell, 1928. Ann. Mag. Nat. Hist., 10(1): 353

Megachile lagopoda var. fulvohirta Alfken, 1935. Veröff. dt. Kolon. u. Ubersee-Mus. Bremen, 1: 191 (non Alfken, 1926)

Megachile furukawai Yasumatsu, 1938. Insecta matsum., 13: 33

Subespecie distribuida en Europa suroccidental, desde España, y, en Argelia en el norte de África.

\section{Megachile (Xanthosarus) lagopoda seitziana Cockerell,} 1925

Megachile lagopoda seitziana Cockerell, 1925. The Entomologist, 58: 159

Endemismo ibérico, sólo conocido de los Pirineos.

Megachile (Xanthosarus) maritima (Kirby, 1802)

Apis maritima Kirby, 1802. Monogr. Apum Angl., 2: 242

Megachile flaviventris Schenck, 1853. Jahrb. Ver. Nat.kd. Nassau, 9: 174

Megachile fulvescens Smith, 1853. Cat. Hymenopt. Insects Collect. Br. Mus., 1: 152 (non Walker, 1871)

Phyllotoma manicata Duméril, 1860. Mém. Acad. Sci. Inst. Fr., 31: 845 (non Megachile manicata Giraud, 1861)

9 Para Tkalcu (1974), Megachile bicoloriventris Mocsáry, 1878 era una especie válida. 
Megachile kashgarensis Cockerell, 1913. Ann. Mag. Nat. Hist., 8(12): 371

Especie distribuida por casi toda Europa y por Marruecos, en el norte de África; llega hasta Kazajistán.

Megachile (Xanthosarus) nigriventris Schenck, 1870

?Megachile ursula Gerstaecker, 1869. Stett. Entomol. Ztg., 30: 355 Megachile nigriventris Schenck, 1870. Jahrb. Ver. Nat.kd. Nassau, 21/22 [1867-1868]: 324

Megachile nigriventris Schenck, 1870. Jahrb. Ver. Nat.kd. Nassau, 21/22 [1867-1868]: 324

Megachile curvicrus Thomson, 1872. Hym. Scand., 2: 223

Megachile hasticornis Cockerell, 1924. Ann. Mag. Nat. Hist., 9(13): 601

Especie europea que presenta una serie de subespecies, de las que sólo la nominal aparece en el territorio mediterráneo occidental (Europa suroccidental). Dos citas ibéricas, una del Pirineo de Huesca reseñada por Ceballos (1956) y Pérez-Íñigo (1984), y otra de Madrid, asimismo de Pérez- Íñigo (1984), deberán ser confirmadas.

Megachile (Xanthosarus) willughbiella (Kirby, 1802) Apis willughbiella Kirby, 1802. Monogr. Apum Angl., 2: 233

Distribuida ampliamente en Europa y en el norte de África. En el área mediterránea occidental presenta dos subespecies.

Megachile (Xanthosarus) willughbiella willughbiella (Kirby, 1802)

Apis willughbiella Kirby, 1802. Monogr. Apum Angl., 2: 233

Megachile atriventris Schenck, 1853. Jahrb. Ver. Nat.kd. Nassau, 9: 178

Megachile centuncularis var. janssoni Alfken, 1926. Ent. Tidskr., 47: 202

Megachile korotnevi Cockerell, 1928. Ann. Mag. Nat. Hist., 10(1): 357

Subespecie europea, repartida desde España hasta Siberia.

Megachile (Xanthosarus) willughbiella zeraldae Tkalců, 1988

Megachile (Delomegachile) willughbiella zeraldae Tkalců, 1988. Vêst. cs. Spolec. zool., 52: 57

Subespecie endémica de Argelia.

\section{Incertae sedis}

Megachile arcigera Pérez, 1895

Megachile arcigera Pérez, 1895. Espèces Nouv. Mellif. Barbarie: 24

Descrita de "Barbarie", aunque Pérez (1895) no aporta datos de localidad concreta, si bien habla de
«Algerie, Tebessa...». No se ha podido corroborar con datos posteriores.

\section{Megachile bioculata Pérez 1902}

Megachile bioculata Pérez 1902. P.-v. Soc. Linn. Bordeaux, 57: 119

Especie descrita de Cataluña, cita que Ceballos (1956) recoge pero de la que no ha vuelto a haber datos posteriores.

\section{Megachile callensis Ferton, 1914}

Megachile callensis Ferton, 1914. Ann. Soc. Entomol. France, 83: 93

Descrita con un solo ejemplar, una hembra de La Calle (Argelia). Posteriormente se describió Megachile callensis var. regularis Benoist, 1934 (Bull. Soc. Entomol. France, 39: 109), a partir de una hembra capturada en Rabat (Marruecos), junto con ejemplares de la forma típica. No se han vuelto a aportar datos de ninguna de las dos, por lo que, sin la localización de los tipos, no se puede confirmar su validez.

\section{Megachile melanota Pérez, 1895}

Megachile melanota Pérez, 1895. Espèces Nouv. Mellif. Barbarie: 23

Descrita de "Barbarie", no se ha vuelto a corroborar con datos posteriores. Según Pérez (1895), es "muy vecina de octosignata", por lo que se emplazaría en el subgénero nominal, a falta de la revisión del tipo.

\section{Megachile mguildensis Benoist, 1940}

Megachile mguildensis Benoist, 1940. Ann. Soc. Entomol. France, 109: 50

Especie descrita con material de Marruecos (Atlas Medio) y también conocida de Argelia, pero de la que no hay citas recientes.

\section{Género Coelioxys Latreille, 1809}

Coelioxys Latreille, 1809. Gen. Crust. Ins., 4: 166 ESPECIE TIPO: Apis conica Linnaeus, 1758 = Apis quadridentata Linnaeus, 1758, por designación de Latreille, 1831

Coelioxys (Paracoelioxys) Gribodo, 1884. Boll. Soc. entomol. Ital., 16: 274

ESPECIE TIPO: Coelioxys montandoni Gribodo, $1884=$ Coelioxys alata Förster, 1853, por monotipia

Coelioxys (Tropicocoelioxys) Gupta, 1991. J. Bomb. Nat. Hist. Soc., 88: 425

EsPECIE TIPO: Coelioxys genoconcavitus Gupta, 1991, por designación original

Coelioxys (Orientocoelioxys) Gupta, 1992. Reichenbachia, 29: 73 ESPECIE TIPO: Coelioxys quadrifasciatus Gupta, 1992, por designación original 
Coelioxys (Nigrocoelioxys) Gupta, 1993. Taxon. Stud. Megach. North-West. India: 235

ESPECIE TIPO: Coelioxys fuscipennis Smith, 1854, por designación original

Género casi cosmopolita que contiene varios cientos de especies y que, probablemente, encuentra su máxima diversidad en Suramérica (Michener, 2000). Si bien la clasificación subgenérica presenta discordancias según que el género sea estudiado desde la óptica del Viejo o el Nuevo Mundo, hay que destacar el esfuerzo de, por ejemplo, Schwarz (1999) o Přidal \& Tkalcù (2001) por aclarar algunas situaciones poco claras. Reflejo de esta situación es que Michener (2000), por ejemplo, no incluyera los subgéneros descritos por Ruszkowski (en Ruszkowski et al., 1986), seguramente por desconocer su trabajo.

\section{Subgénero Allocoelioxys Tkalců, 1974}

Allocoelioxys Tkalců, 1974. Beitr. Ent., 24(5/8): 340

ESPECIE TIPO: Coelioxys afra Lepeletier, 1841, por designación original

Coelioxita Pasteels, 1977. Rev. Zool. Afric., 91: 180

ESPECIE TIPO: Coelioxys afra Lepeletier, 1841, por designación original

Coelioxula Pasteels, 1982. Bull. Ann. Soc. R. Belg. Entomol., 118: 110 (nomen nudum)

Coelioxys (Intercoelioxys) Ruszkowski, 1986. Pzsczelnicze Zesz. nauk., 30: 117

ESPECIE TIPO: Coelioxys rufocaudata Smith, $1854=$ Coelioxys echinata Förster, 1853, por monotipia

Coelioxys (Lepidocoelioxys) Ruszkowski, 1986. Pzsczelnicze Zesz. nauk., 30: 117 (nomen nudum)

Subgénero distribuido por el Viejo Mundo (regiones paleártica, oriental y etiópica). Presenta más de 40 especies: unas 9 europeas, 15 paleárticas occidentales, 21 subsaharianas y unas pocas del sur de Asia (Michener, 2000).

\section{Coelioxys (Allocoelioxys) acanthura (Illiger, 1806)}

Anthophora acanthura Illiger, 1806. Mag. Insektenk., 5: 106 Coelioxys macrura Förster, 1853. Ver. Nat.hist. Ver. Preuss. Rheinl. Westf., 10: 283

Coelioxys italica Gistel, 1857. Vacuna, 2: 543, grafía original incorrecta

Coelioxys undecimdentata Radoszkowski, 1893. Horae Soc. Entomol. Ross., 27 [1892-1893]: 50, grafía original incorrecta

Especie repartida desde España por la región paleártica suroccidental.

\section{Coelioxys (Allocoelioxys) afer Lepeletier, 1841}

Coelioxys afra Lepeletier, 1841. Hist. Nat. Insectes Hyménopt., 2: 525 , grafía original incorrecta

Coelioxys coronata Förster, 1853. Ver. Nat.hist. Ver. Preuss. Rheinl. Westf., 10: 280, grafía original incorrecta
Coelioxys mandibularis Chévrier, 1872. Mitt. schweiz. ent. Ges., 3(10): 487 (non Nylander, 1848)

Coelioxys afra var. tunensis Gribodo, 1894. Boll. Soc. entomol. Ital., 26: 83, grafía original incorrecta

Especie repartida desde España y Portugal por la región paleártica occidental.

\section{Coelioxys (Allocoelioxys) brevis Eversmann, 1852}

Coelioxys brevis Eversmann, 1852. Bull. Soc. Imp. Nat. Moscou, 25: 77

Coelioxys erythropyga Förster, 1853. Ver. Nat.hist. Ver. Preuss. Rheinl. Westf., 10: 286, grafía original incorrecta

Coelioxys erythropyga var. rubida Gribodo, 1894. Boll. Soc. entomol. Ital., 26: 84, grafía original incorrecta

Coelioxys inflatus Alfken, 1933. Mitt. D. Ent. Ges., 4: 135

Distribuida desde la Península Ibérica (España y Portugal) prácticamente por toda la región paleártica occidental.

\section{Coelioxys (Allocoelioxys) caudatus Spinola, 1838}

Coelioxys caudata Spinola, 1838. Ann. Soc. Entomol. France, 7: 535, grafía original incorrecta

Coelioxys försteri Morawitz, 1871. Horae Soc. Entomol. Ross., 8: 211

Especie repartida desde España por la región paleártica occidental.

\section{Coelioxys (Allocoelioxys) coturnix Pérez, 1883}

Coelioxys coturnix Pérez, 1883. Act. Soc. Linn. Bordeaux, 37: 278

Coelioxys taurus Nurse, 1902. J. Asiat. Soc. Beng., 70: 153

Coelioxys ruficaudis Cameron, 1913. Indian forest Rec., 4: 122

Especie distribuida por Francia y España.

\section{Coelioxys (Allocoelioxys) echinatus Förster, 1853}

?Coelioxys octodentata Lepeletier, 1841. Hist. Nat. Insectes Hyménopt., 2: 534 (non Say, 1824) (nomen dubium), grafía original incorrecta

?Coelioxys ruficauda ${ }^{10}$ Lepeletier, 1841. Hist. Nat. Insectes Hyménopt., 2: taf. 14, figs. 2, 3 (nomen dubium)

Coelioxys echinata Förster, 1853. Ver. Nat.hist. Ver. Preuss. Rheinl. Westf., 10: 279, grafía original incorrecta

Coelioxys rufocaudata Smith, 1854. Cat. Hymenopt. Insects Collect. Br. Mus., 2: 260 (nomen novum para Coelioxys octodentata Lepeletier, 1841), grafía original incorrecta

Coelioxys antennalis Pérez, 1883. Act. Soc. Linn. Bordeaux, 37: 282

Coelioxys aegypticola Friese, 1925. Konowia, 4: 33

Especie repartida desde la Península Ibérica por la región paleártica occidental, excepto las zonas más frías.

\footnotetext{
${ }^{10}$ Véase la opinión de Schwarz et al. (1996) en relación con la consideración que de Coelioxys ruficauda Lepeletier, 1841 y Coelioxys obtusa Pérez, 1883 hacía Warncke (1992).
} 
Coelioxys (Allocoelioxys) elongatulus Alfken, 1938

Coelioxys elongatula Alfken, 1938. Dtsch. Entomol. Z., 1938: 429, grafía original incorrecta

Principalmente, la distribución de esta especie es mediterránea oriental, y se extiende por Grecia, Chipre, Creta, Turquía, Israel y Palestina, pero se reparte también por Argelia.

Coelioxys (Allocoelioxys) elytrura Spinola, 1838

Coelioxys elytrura Spinola, 1838. Ann. Soc. Entomol. France, 7: 532

Coelioxys obtusa Pérez, 1883. Act. Soc. Linn. Bordeaux, 37: 279 , grafía original incorrecta

Coelioxys laticauda Morawitz, 1895. Horae Soc. Entomol. Ross., 29: 35

Especie europea suroccidental, repartida por Portugal, España, Francia, Austria y Sicilia. Además, ha sido citada, como C. laticauda, de Turkmenistán.

Schwarz \& Gusenleitner (2003) discutían y establecían que Coelioxys elytrura, auctt. es la especie Coelioxys indica Friese, 1925, de Egipto, la India y otros territorios orientales, y que Coelioxys elytrura Spinola, 1838 es la Coelioxys obtusa de Pérez.

\section{Coelioxys (Allocoelioxys) emarginatus Förster, 1853}

Coelioxys emarginata Förster, 1853. Ver. Nat.hist. Ver. Preuss. Rheinl. Westf., 10: 288, grafía original incorrecta

Coelioxys minuta Gistel, 1857. Vacuna, 2: 548 (non Smith, 1879), grafía original incorrecta

Coelioxys robusta Morawitz, 1875. En: Fedtschenko, Izv. Imp. Obsestva. Lûbit. Estestv. Antropol. Etnogr., 19: 136, grafía original incorrecta

Distribuida en Europa occidental y meridional, desde Francia y España, hasta Asia Central y por Marruecos, en África.

\section{Coelioxys (Allocoelioxys) haemorrhous Förster, 1853}

Coelioxys haemorrhoa Förster, 1853. Ver. Nat.hist. Ver. Preuss. Rheinl. Westf., 10: 285, grafía original incorrecta

Coelioxys pulchella Morawitz, 1874. Horae Soc. Entomol. Ross., 10 [1873-1874]: 187, grafía original incorrecta

Especie distribuida por el sur de Europa, desde la Península Ibérica hasta Austria, y el norte de África, hasta Asia Central.

\section{Coelioxys (Allocoelioxys) polycentris Förster, 1853}

Coelioxys polycentris Förster, 1853. Ver. Nat.hist. Ver. Preuss. Rheinl. Westf., 10: 282

Coelioxys conspersa Morawitz, 1874. Horae Soc. Entomol. Ross., 10 [1873-1874]: 185, grafía original incorrecta

Coelioxys orientalis Friese, 1925. Konowia, 4: 32

Distribuida por Europa central y meridional, incluida Chipre, hasta Daguestán y Mongolia. Unas citas ibéricas referenciadas por Ceballos (1956; tomado de Dusmet, 1906) deberían ser confirmadas.

\section{Subgénero Coelioxys Latreille, 1809}

Subgénero holártico que contiene 50 especies en el Viejo Mundo (Michener, 2000).

\section{Coelioxys (Coelioxys) aurolimbatus Förster, 1853}

Coelioxys aurolimbata Förster, 1853. Ver. Nat.hist. Ver. Preuss. Rheinl. Westf., 10: 298, grafía original incorrecta

Coelioxys apiculata Förster, 1853. Ver. Nat.hist. Ver. Preuss. Rheinl. Westf., 10: 290 (non Nylander, 1848), grafía original incorrecta

Coelioxys recurva Schenck, 1853. Jahrb. Ver. Nat.kd. Nassau, 9: 201, grafía original incorrecta

Coelioxys reflexa Schenck 1855. Jahrb. Ver. Nat.kd. Nassau, 10: 147, grafía original incorrecta

Coelioxys ogivalis Pérez, 1895. Espèces Nouv. Mellif. Barbarie: 25 Coelioxys aurolimbata var. algeriensis Friese, 1895. Bienen Eur., 1: 54, grafía original incorrecta

Distribución, desde España, prácticamente paleártica occidental.

\section{Coelioxys (Coelioxys) conoideus (Illiger, 1806)}

Apis conica var. $\gamma$-minor Kirby, 1802. Monogr. Apum Angl., 2: 226

Anthophora conoidea Illiger, 1806. Mag. Insektenk., 5: 105

Coelioxys vectis Curtis, 1831. British Entomol., 8: 349

Coelioxys punctata Lepeletier, 1841. Hist. Nat. Insectes Hyménopt., 2: 520, grafía original incorrecta

Coelioxys temporalis Nylander, 1848. Not. Sällsk. Fauna Flora Fenn. Förh.,1 (Adnot): 253

Coelioxys ambigua Schenck, 1855. Jahrb. Ver. Nat.kd. Nassau, 10: 143, grafía original incorrecta

Coelioxys aegyptiaca Radoszkowski, 1876. Horae Soc. Entomol. Ross., 12(2): 118, grafía original incorrecta

Coelioxys spissicauda Pasteels, 1968. Ann. Mus. R. Afriq. Centr., Sci. Zool., 167: 68

Especie repartida desde la Peninsula Ibérica (España y Portugal) por la región paleártica, a excepción de la parte más septentrional y el extremo oriental.

\section{Coelioxys (Coelioxys) elongatus Lepeletier, 1841}

Coelioxys elongata Lepeletier, 1841. Hist. Nat. Insectes Hyménopt., 2: 522, grafía original incorrecta

Coelioxys simplex Nylander, 1852. Not. Sällsk. Fauna Flora Fenn. Förh., 2 (Revis.): 279 (non Gmelin, 1790)

Coelioxys tricuspidata Förster, 1853. Ver. Nat.hist. Ver. Preuss. Rheinl. Westf., 10: 302, grafía original incorrecta

Coelioxys denticulata Schenck, 1855. Jahrb. Ver. Nat.kd. Nassau, 10: 142, grafía original incorrecta

Coelioxys stigmatica Schenck, 1855. Jahrb. Ver. Nat.kd. Nassau, 10: 142, grafía original incorrecta

Coelioxys distincta Schenck, 1855. Jahrb. Ver. Nat.kd. Nassau, 10: 147, grafía original incorrecta

Coelioxys sponsa Smith, 1855. Cat. Hymenopt. Insects Collect. Br. Mus., 1: 147, grafía original incorrecta 
Coelioxys tridenticulata Schenck, 1861. Jahrb. Ver. Nat.kd. Nassau, 14 [1859]: 372, grafía original incorrecta

Coelioxys gracilis Schenck, 1861. Jahrb. Ver. Nat.kd. Nassau, 14 [1859]: 373

Coelioxys obscura Schenck, 1861. Jahrb. Ver. Nat.kd. Nassau, 14 [1859]: 377, grafía original incorrecta

Coelioxys claripennis Schenck, 1870. Jahrb. Ver. Nat.kd. Nassau, 21/22 [1867-1868]: 353

Coelioxys kudiana Cockerell, 1924. Ann. Mag. Nat. Hist., 9(13): 524, grafía original incorrecta

Coelioxys popovici Friese, 1925. Konowia, 4: 31

Especie repartida desde España y Portugal por la región paleártica occidental, a excepción de la parte más septentrional.

\section{Coelioxys (Coelioxys) inermis (Kirby, 1802)}

Apis centuncularis acuminata Christ, 1791. Nat.gesch. Classif. Nomencl. Insekten: 192 (non Gmelin, 1790)

Apis inermis Kirby, 1802. Monogr. Apum Angl., 2: 229

Coelioxys decempunctata Spinola, 1838. Ann. Soc. Entomol. France, 7: 533, grafía original incorrecta

Coelioxys acuminata Nylander, 1852. Not. Sällsk. Fauna Flora Fenn. Förh., 2 (Revis.): 279 (non Gmelin, 1790; nec Christ, 1791)

Coelioxys microdonta Förster, 1853. Ver. Nat.hist. Ver. Preuss. Rheinl. Westf., 10: 291, grafía original incorrecta

Coelioxys divergens Förster, 1853. Ver. Nat.hist. Ver. Preuss. Rheinl. Westf., 10: 292

Distribuida desde España, prácticamente por toda la región paleártica, a excepción de su parte más septentrional y el extremo suroriental.

\section{Coelioxys (Coelioxys) lanceolatus Nylander, 1852}

Coelioxys lanceolata Nylander, 1852. Not. Sällsk. Fauna Flora Fenn. Förh., 2 (Revis.): 279, grafía original incorrecta Coelioxys patula Pérez, 1883. Act. Soc. Linn. Bordeaux, 37: 297, grafía original incorrecta

Distribuida por Europa, a excepción de su parte más meridional (llega sólo a los Pirineos y los Alpes).

\section{Coelioxys (Coelioxys) mandibularis Nylander, 1848}

Coelioxys mandibularis Nylander, 1848. Not. Sällsk. Fauna Flora Fenn. Förh., 1 (Adnot.): 252

Distribuida por Europa, desde los Pirineos (pero ausente de las penínsulas Ibérica e Italiana) e incluida Gran Bretaña, hasta Mongolia; Warncke $(1988,1992)$ rechazaba citas anteriores de Argelia, en el norte de África.

Coelioxys (Coelioxys) osmiae Alfken, 1928

Coelioxys osmiae Alfken, 1928. Konowia, 7: 193

Especie endémica de Túnez.

Coelioxys (Coelioxys) quadridentatus (Linnaeus, 1758) Apis quadridentata Linnaeus, 1758. Syst. Nat., ed. 10, 1: 577
Apis conica Linnaeus, 1758. Syst. Nat., ed. 10, 1: 578

?Apis agilis Harris, 1776. Expos. English. Insects:163, Tab. 49, Fig. 8

Apis acuminata Gmelin, 1790. Linné Syst. Nat., ed.13, 1(5): 2789

Apis bidentata Panzer, 1798. Faunae Insector. Ger., 59: $\mathrm{n}^{\mathrm{o}} 7$

Coelioxys acuta Nylander, 1848. Not. Sällsk. Fauna Flora Fenn. Förh., 1 (Adnot): 250, grafía original incorrecta

Coelioxys fissidens Förster, 1853. Ver. Nat.hist. Ver. Preuss. Rheinl. Westf., 10: 293

Coelioxys fraterna Förster, 1853. Ver. Nat.hist. Ver. Preuss. Rheinl. Westf., 10: 294, grafía original incorrecta

Coelioxys convergens Schenck, 1855. Jahrb. Ver. Nat.kd. Nassau, 10: 147

Coelioxys truncata Hoppner, 1901. Abh. naturw. Ver. Bremen, 15: 252, grafía original incorrecta

Distribución europeo-anatólica, desde España y Portugal e incluida Gran Bretaña, hasta Siberia.

Coelioxys (Coelioxys) rufescens Lepeletier \& Serville, 1825

Coelioxys rufescens Lepeletier \& Serville, 1825. Encycl. Méthod., 10 (Insectes): 109

Coelioxys umbrina Smith, 1843. Zoologist, 1: 63, grafía original incorrecta

Coelioxys hebescens Nylander, 1848. Not. Sällsk. Fauna Flora Fenn. Förh., 1 (Adnot.): 251

Coelioxys apiculata Nylander, 1848. Not. Sällsk. Fauna Flora Fenn. Förh., 1 (Adnot.): 282, grafía original incorrecta Coelioxys diglypha Förster, 1853. Ver. Nat.hist. Ver. Preuss. Rheinl. Westf., 10: 295, grafía original incorrecta

Coelioxys trinacria Förster, 1853. Ver. Nat.hist. Ver. Preuss. Rheinl. Westf., 10: 300

Coelioxys obtusata Schenck, 1855. Jahrb. Ver. Nat.kd. Nassau, 10: 144 (non Magretti, 1895), grafía original incorrecta

Coelioxys carinata Schenck, 1855. Jahrb. Ver. Nat.kd. Nassau, 10: 147 (non Smith, 1854), grafía original incorrecta

Coelioxys parvula Schenck, 1855. Jahrb. Ver. Nat.kd. Nassau, 10: 147, grafía original incorrecta

Coelioxys longiuscula Schenck, 1855. Jahrb. Ver. Nat.kd. Nassau, 10: 148, grafía original incorrecta

Coelioxys fallax Mocsáry, 1881. Termeszettud. Közl.,16: 67

Especie paleártica occidental, que en Europa se reparte desde España y en África noroccidental ha sido citada en Marruecos y Argelia.

\section{Subgénero Liothyrapis Cockerell, 1911}

Coelioxys (Liothyrapis) Cockerell, 1911. Proc. U. S. Nat. Mus., 40: 246

ESPECIE TIPO: Coelioxys apicata Smith, $1854=$ Coelioxys decipiens Spinola, 1838, por designación original

Coelioxys (Liothgrapis) Cockerell, 1932. Rev. Zool. Bot. Afric., 23: 26, grafía posterior incorrecta

Liothgraphis Sandhouse, 1943. Proc. U.S. Nat. Mus., 92: 564, grafía posterior incorrecta de Liothgrapis Cockerell, 1932

Coelioxys (Hemicoelioxys) Pasteels, 1968. Ann. Mus. R. Afriq. Centr., Sci. Zool., 167: 133

ESPECIE TIPO: Coelioxys gracilis Pasteels, 1968 (non Schenck, 1861) = Hemicoelioxys gracillima Pasteels, 1977, por designación original 
Subgénero presente en toda África (excepto el Sahara) y el área mediterránea oriental, y que se extiende hacia el este por la región transcaspiana y el sur de Asia. Se estima que existen en torno a 35 especies (Michener, 2000).

Schwarz (1999, 2001) degradó Hemicoelioxys Pasteels, 1968 a subgénero de Coelioxys Latreille, 1809 y estableció que Hemicoelioxys Pasteels, 1968 sensu Gupta (1993), es sinónimo de Radoszkowskiana Popov, 1955.

\section{Coelioxys (Liothyrapis) decipiens Spinola, 1838}

Coelioxys decipiens Spinola, 1838. Ann. Soc. Entomol. France, 7: 532

Coelioxys cretensis Förster, 1853. Ver. Nat.hist. Ver. Preuss. Rheinl. Westf., 10: 276

Coelioxys farinosa Smith, 1854. Cat. Hymenopt. Insects Collect. Br. Mus., 2: 260, grafía original incorrecta

Dioxys albofasciata Radoszkowski, 1888. Horae Soc. Entomol. Ross., 22: 340, grafía original incorrecta

Coelioxys seraxensis Radoszkowski, 1893. Horae Soc. Entomol. Ross., 27 [1892-1893]: 52

Coelioxys aberrans Morawitz, 1895. Horae Soc. Entomol. Ross., 29: 33

Coelioxys decemdentata Pérez, 1902. P.-v. Soc. Linn. Bordeaux, 57: 121, grafía original incorrecta

Coelioxys proxima Friese, 1925. Konowia, 4: 31 (non Holmberg, 1916), grafía original incorrecta

Coelioxys proximata Popov, 1946. Proc. R. Ent. Soc. London (B), 15: 109 (nomen novum para Coelioxys proxima Friese, 1925), grafía original incorrecta

Coelioxys sinuata Stanek, 1968. Bull. Rech. agron. Gembloux, 3: 371, grafía original incorrecta

Especie distribuida por todo el norte de África, Chipre, Creta y Turquía, Asia Menor y Asia Central hasta el Himalaya.

\section{Subgénero Mesocoelioxys Ruszkowski, 1986}

Coelioxys (Mesocoelioxys) Ruszkowski, 1986. Pzsczelnicze Zesz. nauk., 30: 117

ESPECIE TIPO: Coelioxys argentea Lepeletier, 1841, por monotipia

Argcoelioxys Warncke, 1992. Ber. Naturf. Ges. Augsburg, 53: 39

ESPECIE TIPO: Coelioxys argentea Lepeletier, 1841, por designación original

Distribución mediterráneo-turano-centroasiática. El subgénero sólo incluye una especie, que presenta caracteres muy próximos a los del subgénero nominal, en el que, según Michener (2000), podría llegar a incluirse en el caso de realizarse una revisión global del género. Hay que indicar que ese autor desconocía la descripción del subgénero, y un trabajo posterior (Přidal \& Tkalců, 2001) ha rehabilitado su uso.

Coelioxys (Mesocoelioxys) argenteus Lepeletier, 1841

Coelioxys argentea Lepeletier, 1841. Hist. Nat. Insectes Hyménopt., 2: 523, grafía original incorrecta
Coelioxys constricta Förster, 1853. Ver. Nat.hist. Ver. Preuss. Rheinl. Westf., 10: 274, grafía original incorrecta

Coelioxys diplotaenia Förster, 1853. Ver. Nat.hist. Ver. Preuss. Rheinl. Westf., 10: 277

Coelioxys transcaspica Radoszkowski, 1886. Horae Soc. Entomol. Ross., 20 [1885-1887]: 17, grafía original incorrecta

La distribución de esta especie coincide con la del subgénero, es decir, se extiende desde España y Portugal por el territorio mediterráneo-turano-centroasiático.

\section{Comentario final y conclusiones}

Tal como se señaló en la primera parte de este estudio (Ornosa et al., 2006), la naturaleza de un trabajo como éste hace difícil extraer conclusiones distintas del propio contenido global del catálogo en sí mismo, pero a modo de resumen puede establecerse que se han rastreado, revisado y consignado todos los taxones de Lithurgini y Megachilini del territorio comprendido en la región occidental de la cuenca mediterránea, hasta el nivel de subespecie, sus sinonimias y citas, desde cada descripción original -salvo muy raras excepciones- hasta las más recientes. El total es de 5 géneros, 16 subgéneros, 106 especies y 126 subespecies.

En relación con su distribución geográfica (Tabla 1), los géneros que constituyen las tribus Lithurgini y Megachilini en el área mediterránea occidental tienen una amplia dispersión, predominando los subcosmopolitas. Atendiendo al patrón corológico por especies (Tabla 2), empleando las categorías clásicas actualizadas por La Greca (1990) y Vigna-Taglianti et al. (1999), se observa que, aunque existen algunas holárticas y paleárticas (que, en conjunto, alcanzan el $4 \%$ del total), muestran un mayor porcentaje el conjunto de elementos

Tabla 1.- Distribución geográfica de los géneros de Lithurgini y Megachilini.

Table 1.- Geographical distribution of the genera of Lithurgini and Megachilini.

\begin{tabular}{lc}
\hline GÉNERO & DISTRIBUCIÓN GEOGRÁFICA \\
\hline Lithurgus & Cosmopolita \\
Creightonella & Paleártica y Etiópica \\
Chalicodoma & Subcosmopolita \\
Megachile & Subcosmopolita \\
Coelioxys & Subcosmopolita \\
\hline
\end{tabular}


Tabla 2.- Principales corotipos que existen en las tribus Lithurgini y Megachilini (categorías según La Greca, 1990 y Vigna-Taglianti et al., 1999).

Table 2.- Main chorotypes in tribes Lithurgini and Megachilini (categories after La Greca, 1990 and Vigna-Taglianti et al., 1999).

\begin{tabular}{lc}
\hline PATRÓN COROLÓGICO & \% \\
\hline Holártica & 1 \\
Paleártica & 3 \\
Paleártica occidental & 23 \\
Europea & 19 \\
Circunmediterránea & 8 \\
Mediterránea occidental & 8 \\
Mediterráneo-turánica & 7 \\
Norte de África & 16 \\
Íbero-magrebí & 0 \\
Endemismos ibéricos & 4 \\
Endemismos no ibéricos & 11 \\
\hline
\end{tabular}

paleárticos occidentales y europeos $(23 \%$ y $19 \%$, respectivamente), seguidos por los corotipos norteafricanos (16\%); en menor proporción aparecen los patrones circunmediterráneos, mediterráneos occidentales y mediterráneo-turánicos $(8 \%, 8 \%$ y $7 \%$, respectivamente), y finalmente, son minoritarios también los endemismos ibéricos (4\%). A nivel especie, no se han encontrado elementos íberomagrebíes. El heterogéneo conjunto que convencionalmente reúne los endemismos no ibéricos da un total del $11 \%$.

De cualquier modo, los estudios taxonómicos en curso sobre los integrantes de la familia en el territorio estudiado permitirán en el futuro hacer afirmaciones biogeográficas más concluyentes.

\section{AGRADECIMIENTOS}

A los Doctores Maximilian Schwarz (Ansfelden, Austria) y Peter Hartmann (Universidad de Bayreuth, Alemania), por su opinión ante ciertas dudas taxonómicas y a Mr. Gérard Le Goff (Barentin, Francia), por poner a la disposición de los autores una amplia información sobre material de megaquílidos de España. Ernst-Gerhard Burmeister ha suministrado información relativa a las fechas de publicación de ciertos números del Nachrichtenblatt der Bayerischen Entomologen. Miguel Ángel Alonso-Zarazaga, uno de los evaluadores de este trabajo, ha detectado la necesidad del cambio de género gramatical de Coelioxys a masculino, a diferencia del sentido tradicional que le han dado los autores, y la consiguiente adecuación de la terminación de los taxones que así lo han requerido.

\section{Referencias}

Alfken, J. D., 1914. Beitrag zur Kenntnis der Bienenfauna von Algerien. Memoires de la Société Entomologique de Belgique, 22 [1913-1914]: 185237.

AlfKen, J. D., 1924. Megachile centuncularis L. und ihre Verwandten. Deutsche Entomologische Zeitschrift, 1924: 355-360.

AlfKen, J. D., 1931. Über das Walliser "Chalicodoma baeticum" (Hym. Apid.). Mitteilungen der Deutsche Entomologischen Gesellschaft, 2: 18-19.

AlfKen, J. D., 1932. Beitrag zur Kenntnis der Chalicodoma-Arten von Ägypten. Bulletin de la Société Royale entomologique d'Egypte, 1932: 223230.

AlfKen, J. D., 1938a. Ein weiterer Beitrag zur Kenntnis Bienenfauna von Palästina mit Einschluss des SinaiGebirges (Hym. Apid.). Deutsche Entomologische Zeitschrift,1938: 418-433.

AlfKen, J. D., 1938b. Contributi alla conoscenza della fauna entomologica della Sardegna. Apidae. Memorie della Società Entomologica Italiana, 16: 97-114.

AlfKen, J. D., 1941. Welchen wissenschaftlichen Namen hat die schwarze Mörtelbiene zu führen? (Hym. Apid.). Mitteilungen der Münchener Entomologischen Gesellschaft, 31: 89-92.

AlfKen, J .D. \& Bischoff, H., 1933. Ueber die von Erichson in "Waltl, Reise durch Tyrol, Oberitalien und Piemont nach dem südlichen Spanien" beschriebenen Bienen. Sitzungsberichte der Gesellschaft naturforschender Freunde, 1932: 508-514.

BAKER, D. B., 1993. The type material of the nominal species of exotic bees described by Frederick Smith. $\mathrm{Ph}$. D. Thesis, Oxford Universtity. Oxford. vi + 312 pp.

BANASZAK, J. \& Romasenko, L., 2001. Megachilid bees of Europe (Hymenoptera, Apoidea, Megachilidae). Pedagogical University of Bydgoszcz. Bydgoszcz. 239 pp.

BENOIST, R., 1924. Sur la provenance de quelques Hyménoptères Mellifères décrits par J. Pérez. Bulletin de la Société entomologique de France, 29: 109-111.

Benoist, R., 1934. Descriptions d'espèces nouvelles de Hyménoptères Mellifères. Bulletin de la Société entomologique de France, 39: 106-110.

BENOIST, R., 1935. Remarques sur quelques espèces du genre Megachile (Hymen. Apidae). Annales de la Société entomologique de France, 104: 97-108.

BENOIST, R., 1940. Remarques sur quelques espèces de Mégachiles principalement de la faune française (Hymen. Apidae). Annales de la Société entomologique de France, 109: 41-88. 
BENOIST, R., 1950. Hyménoptères récoltés par une mission suisse au Maroc (1947). Apidae, Megachilinae. Bulletin de la Société des Sciences Naturelles du Maroc, 30: 183-193.

BENOIST, R., 1969. Contribution à la connaissance de la faune des Apides de l'Afrique du Nord et de l'Arabie (Hym). Bulletin de la Société entomologique de France, 74: 243-247.

Ceballos, G., 1956. Catálogo de los Himenópteros de España. Trabajos del Instituto Español de Entomología. (C.S.I.C.). Madrid. 554 pp.

Ceballos, G., 1959. Primer suplemento al Catálogo de los Himenópteros de España. Eos, 35: 217-242.

CurTis, J. (ed.), 1828. British Entomology: Being illustrations and descriptions of the genera of Insects found in Great Britain and Ireland. 5. London. Lams. 195-241.

Diniz, M. A., 1960a. Notas sobre himenópteros de Portugal, I. Memórias e Estudos do Museu Zoológico da Universidade de Coimbra, 266: 1-37.

DinIZ, M. A., 1960b. Estado actual do conhecimento dos himenópteros portugueses. Las Ciencias, 25: 211-224.

Diniz, M. A., 1989. Catálogo das abelhas portuguesas I. Ciências Biológicas Ecological Systems (Portugal), 9(1/2): 33-39.

Dusmet, J. M., 1906. Los Ápidos de España. II. Género Coelioxys. Boletín de la Real Sociedad española de Historia Natural, 6: 134-151.

Dusmet, J. M., 1915a. Ápidos de Marruecos de los Gén. Anthidium, Nomada, Melecta, Crocisa, Coelioxys y Phiarus. Memorias de la Real Sociedad española de Historia Natural, 8: 293-336.

Dusmet, J. M., 1915b. Nota sobre los Ápidos de Marruecos. Boletín de la Real Sociedad española de Historia Natural, 15: 255-258.

Dusmet, J. M., 1921. Contribución al conocimiento de los himenópteros de Portugal: lista de los cazados en junio de 1921. Asociación española para el Progreso de las Ciencias, Congreso de Oporto (1921) 6 (4, Ciencias Naturales): 183-191.

Dusmet, J. M., 1931. Contribución al estudio de los Himenópteros de Portugal. Memórias e Estudos do Museu Zoológico da Universidade de Coimbra, Série 1, 52: 1-9.

EBMER, A. W., 2001. Hymenopterologische Notizen aus Österreich - 14 (Insecta: Hymenopteera: Apoidea). Linzer Biologische Beiträge, 33(1): 435-460.

ENGEL, M. S., 2001. A monograph of the Baltic amber bees and evolution of the Apoidea (Hymenoptera). Bulletin of the American Museum of Natural History, 259: 1-192.

ENGEL, M. S., 2002. Phylogeny of the bee tribe Fideliini (Hymenoptera: Megachilidae), with the description of a new genus from southern Africa. African Entomology, 10(2): 305-313.
ENGEL, M. S., 2004. Fideliine phylogeny and classification revisited (Hymenoptera: Megachilidae). Journal of the Kansas Entomological Society, 77(4): 821-836.

ERLANDSSON, S., 1991. Hymenoptera Aculeata from the European part of the Mediterranean countries, IV. Bolletino del Museo civico di Venezia, 40 [1989]: 89-95.

Finnamore, A. T. \& Michener, C. D., 1993. Superfamily Apoidea. En: H. Goulet \& J.T. Huber (eds.), Hymenoptera of the world: An identification guide to families. Centre for Land and Biological Resources Research. Ottawa, Ontario: 279-357.

FrIESE, H., 1895. Die Bienen Europa's (Apidae europaeae) Theil I. Schmarotzerbienen. Friedländer \& Sohn. Berlin. 218 pp.

FRIESE, H., 1898. Species aliquot novae vel minus cognitae generis Megachile Latr. (et Chalicodoma Lep.). Természetrajzi Füzetek, 21: 198-202.

Friese, H., 1899. Die Bienen Europa's (Apidae europaeae) Theil V. Solitäre Apiden. Genus Lithurgus, Genus Megachile (Chalicodoma). C. Lampe. Innsbruck. 228 pp.

Friese, H., 1904. Die Kegelbienen Africa's. (Genus Coelioxys-Hym.). Arkiv för Zoologi, 2(6): 1-16.

FrIESE, H., 1911. Apidae I. Megachilinae. Das Tierreich. Lieferung 28: xxvi +440 pp. Friedländer Berlin.

FrIESE, H., 1925. Neue Formen von Schmarotzerbienen, besonders aus dem paläarktischen Gebiet. Konowia, 4: 27-42.

Gauld, I. \& Bolton, B. (eds.), 1988. The Hymenoptera. British Museum (Natural History). Oxford University Press, London. xi +332 pp.

Gogala, A., 1998. The identity of Megachile dorsalis Pérez and Megachile burdigalensis Benoist, sp. rev. (Hymenoptera: Apoidea: Megachilidae). Acta Entomologica Slovenica, 6(2): 79-87.

GuptA, R. K., 1993. Taxonomic studies on the Megachilidae of north-western India (Insecta, Hymenoptera, Apoidea). Indian Council of Agricultural Research. New Delhi (India). iii + 288 pp.

Heras, C. \& Gayubo, S .F., 1989. Apidofauna de la provincia de Zamora. II. Megachilidae (Hymenoptera, Apoidea). Boletín de la Asociación española de Entomología, 13: 237-249.

Hohmann, H., La Roche, F., Ortega, G. \& Barquín, J., 1993. Bienen, Wespen und Ameisen der Kanarischen Inseln (Insecta: Hymenoptera: Aculeata). Veröffentlichungen aus dem Ubersee-Museum Bremen (Naturwissenschaften), 12(I-II): 14-712.

HuRD, P., 1967. The identity of Megachile rotundata (Fabricius) and M. argentata (Fabricius) (Hymenoptera: Apoidea). Entomologiske Meddelelser, 35: 3-10.

LA GrecA, M., 1990. The Insect Biogeography of West Mediterranean Islands. International Symposium on Biogeographical aspects of insularity. Accademia Nazionale dei Lincei. Roma: 469-491. 
LiOngO LI ENKulu, M., 1988. Les Megachiles (Hymenoptera, Apoïdea) d'Europe et d'Afrique. Une étude écologique et agronomique. Thèse de Doctorat, Faculté des Sciences Agronomiques de 1'Etat. Gembloux. 247 pp. +2 annexes.

McGinley, R. J. \& Rozen, J. G., 1987. Nesting biology, immature stages, and phylogenetic placement of the Palaearctic bee Pararhophites (Hymenoptera: Apoidea). American Museum Novitates, 2903: 1-21.

Michener, C. D., 1951. Superfamily Apoidea. En: C.F.W. Muesebeck \& K.V. Krombein (eds.), Hymenoptera of America North of Mexico - Synoptic Catalog. U.S.D.A. Monograph no. 2. Washington: 1043-1255.

MichenER, C. D., 1979. Biogeography of the bees. Annals of the Missouri Botanical Garden, 66(3): 277-347.

Michener, C. D., 1986. Family-group names among bees. Journal of the Kansas Entomological Society, 59(2): 219-234.

Michener, C. D., 2000. The bees of the World. The Johns Hopkins University Press. Baltimore and London. viii + 913 pp.

Müller, A. \& BAnsac, N., 2004. A specialized pollenharvesting device in western palaearctic bees of the genus Megachile (Hymenoptera, Apoidea, Megachilidae). Apidologie, 35: 329-337.

Ornosa, C. \& Ortiz-SÁnchez, F. J., 2004. Hymenoptera, Apoidea I. En: Fauna Ibérica, vol. 23. Ramos, M.A. et al., (eds.). Museo Nacional de Ciencias Naturales. CSIC. Madrid. 556 pp.

Ornosa, C., Torres, F. \& Ortiz-SÁnchez, F. J., 2006. Catálogo de los Megachilidae del Mediterráneo occidental (Hymenoptera, Apoidea). I. Osmiini. Graellsia, 62(2): 223-260.

Ortiz-SÁnchez, F. J., Ornosa, C. \& Torres, F., 2007. Consideraciones taxonómicas sobre Lithurgus sublaevis Pérez 1897 y Lithurgus chrysurus Fonscolombe 1834 (Hymenoptera, Apoidea, Megachilidae). Linzer Biologische Beiträge, 39(1): 111-116.

Pasteels, J. J., 1965. Révision des Megachilidae (Hymenoptera Apoidea) de l'Afrique Noire, 1. Les Genres Creightoniella [sic!], Chalicodoma et Megachile (s. str.). Annales Musée Royal de l'Afrique Central, Sciences Zoologiques, 137: ix + 579 pp.

PAsteEls, J. J., 1966. Megachilidae (Genres: Creightoniella [sic!], Megachile et Chalicodoma) peu connues ou nouvelles des régions Paléarctique et Africaine. Bulletin et Annales de la Société Royale d'Entomologie de Belgique, 102(1): 1-19.

PÉrez, J., 1879. Contribution à la fauna des Apiaires de France. Première partie. Actes de la Société Linnéenne de Bordeaux, 33: 119-229.

PÉrez, J., 1890. Catalogue des Mellifères du Sud-Ouest. Actes de la Société Linnéenne de Bordeaux, 44: 133200.
PÉREZ, J., 1895. Espèces nouvelles de Mellifères de Barbarie (Diagnoses préliminaires). Gounouilhou. Bordeaux. $64 \mathrm{pp}$.

PÉreZ, J., 1897. Quelques espèces de Mégachiles nouvelles ou mal connues. Procés verbaux de la Société Linnéenne de Bordeaux, 52: 58-67.

PÉrez-Íñigo, C., 1984. Los Ápidos de la Sierra de Guadarrama II. Fam. Andrenidae y Megachilidae (Hym., Apoidea). Graellsia, 39 [1983]: 103-126.

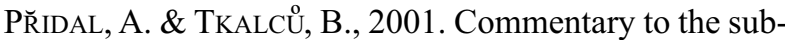
genera of the genus Coelioxys described by Ruszkowski 1986 (Hymenoptera: Apoidea: Megachilidae). Entomofauna, 22(14): 357-364.

Quaranta, M., Ambroselli, S., Barro, P., Bella, S., Carini, A., Celli, G., Cogoi, P., Comba, L., Comoli, R., Felicioli, A., Floris, I., InTOPPA, F., Longo, S., Maini, S., Manino, A., Mazzeo, G., Medrzycki, P., Nardi, E., Niccolini, L., Palmieri, N., Patetta, A., Piatti, C., Piazza, M. G., Pinzauti, M., Porporato, M., Porrini, C., Ricciardelli D’Albore, G., Romagnoli, F., Ruiu, L., SATta, A. \& Zandigiacomo, P., 2004. Wild bees in agroecosystems and semi-natural landscapes. 1997-2000 collection period in Italy. Bulletin of Insectology, 57(1): 11-61.

Rasmont, P., EBmer, A. W., BAnAsZaK, J. \& ZANDEN, G. VAN DER, 1995. Hymenoptera Apoidea Gallica. Bulletin de la Société entomologique de France, 100 (hors série): 1-98.

RoBERTS, R. B., 1978. The nesting biology, behavior and immature stages of Lithurgus chrysurus, an adventitious wood-boring bee in New Jersey. Journal of the Kansas Entomological Society, 51: 735-745.

Roig-Alsina, A. \& Michener, C. D., 1993. Studies of the phylogeny and classification of long-tongued bees (Hymenoptera: Apoidea). The University of Kansas Science Bulletin, 55 (4-5): 123-173.

Ruszkowski, A., Biliński, M. \& GoseK, J., 1986. Ro;liny pokarmowe i gospodarze pasoGytniczych pszczól miesiarkowatych (Coelioxys Latr., Stelis Pz., Dioxys Lep. et Serv., Dioxoides Pop. oraz Paradioxys Mocs. - Hymenoptera, Apidae). Pszczelnicze Zeszyty Naukowe, 30: 111-132.

SchwarZ, M., 1999. Bemerkungen, Korrekturen und Ergänzungen zu den von Gupta (1993) 1999 in "Taxonomic Studies on the Megachilidae of NorthWestern India" behandelten Coelioxys-Arten (Hymenoptera, Apidae). Entomofauna, 20(8): 145 162.

Schwarz, M., 2001. Revision der Gattung Radoszkowskiana Popov 1955 und ein Beitrag zur Kenntnis der Gattung Coelioxys Latreille 1809 (Hymenoptera: Apidae: Megachilinae). Linzer Biologische Beiträge, 33(2): 1267-1286.

SChWARZ, M. \& GuSEnLEITNER, F., 2003. Ergebnisse der Untersuchung von F. Morawitz beschriebener Coelioxys-Arten, so wie weiterer von Eversmann, 
Friese und Radoszkowski beschriebener Arten, nebst einigen Bemerkungen (Hymenoptera: Apidae: Megachilinae). Linzer Biologische Beiträge, 35(2): 1221-1239.

Schwarz, M., Gusenleitner, F., Westrich, P. \& Dathe, H. H., 1996. Katalog der Bienen Österreichs, Deutschlands und der Schweiz (Hymenoptera, Apidae). Entomofauna, Supplement 8: 1-398.

TKALCŮ, B., 1967. Bemerkungen zur Taxonomie einiger paläarktischer Arten der Familie Megachilidae (Hymenoptera, Apoidea). Acta entomologica bohemoslovaca, 64: 91-104.

TKALCŮ, B., 1970. Beiträge zur Kenntnis der Fauna Afghanistans. Chalicodoma Lep., Megachilidae, Apoidea, Hym. Acta Musei Moraviae, 54 (Suppl.) [1969]: 347-384.

TKALCŮ, B., 1974. Ergebnisse der Albanien Expedition 1961 des "Deutschen Entomologischen Institutes". 89. Beitrag. Hymenoptera: Apoidea V (Megachilidae). Beiträge zur Entomologie, 24(5-8): 323-348.

TKALCŮ, B., 1975. Sammelergebnisse der von RNDr. A. Hoffer geleiteten Algerien-Expeditionen in den Jahren 1971 und 1972 (Hymenoptera: Apoidea). 1. Teil: Megachilidae. Acta Rerum Naturalium Musei Nationalis Slovaci, Bratislava, 21: 165-190.

TKALCŮ, B., 1977. Taxonomisches zu einigen paläarktischen Bienenarten (Hymenoptera: Apoidea). Vestnik Ceskoslovenské Spolecnosti Zoologické, 41(3): 223239.

TKALCŮ, B., 1988. Neue paläarktische Arten und Unterarten der Gattungen Chalicodoma und Megachile (Hymenoptera, Apoidea, Megachilidae). Vestnik Ceskoslovenské Spolecnosti Zoologické, 52: 48-62.

Vigna-Taglianti, A., Audisio, P. A., Brondi, M., Bologna, M. A., Carpaneto, G. M., De Biasse, A., Fattorini, S., Piatella, E., Sindaco, R., Venchi, A. \& Zapparoli, M., 1999. A proposal for a chorotype classification of the Near East fauna, in the framework of the Western Palearctic region. Biogeographia, 20 : 31-59.

WARnCKE, K., 1979. Beitrag zur Bienenfauna des Iran 11. Die Gattung Pararhophites Fr. Bolletino del Museo civico di Venezia, 30 : 197-198.

WARnCKE, K., 1980. Fidelia, eine für die Westpaläarktis neue Bienengattung (Hymenoptera, Apidae). Mitteilungen der Münchner Entomologischen Gesellschaft, 70: 89-94.

WARnCKE, K., 1981. Beitrag zur Bienenfauna des Iran 13. Die Bienengattung Lithurgus. Bolletino del Museo civico di Venezia, [1980], 31: 197-199.

WARNCKE, K., 1986. Die Wildbienen Mitteleuropas, ihre gültigen Namen und ihre Verbreitung (Insecta: Hymenoptera). Entomofauna, Supplement 3: 1-128.
WARNCKE, K., 1988. Isolierte Bienenvorkommen auf dem Olymp in Griechenland (Hymenoptera, Apidae). Linzer Biologische Beiträge, 20(1): 83-117.

WARNCKE, K., 1992. Die westpaläarktischen Arten der Bienengattung Coelioxys Latr. (Hymenoptera, Apidae, Megachilinae). Bericht der Naturforschenden Gesellschaft Augsburg, 53: 31-77.

ZANDEN, G. VAN DER, 1977. Notes on some palearctic species of the genus Lithurge Latreille (Hymenoptera Apoidea Megachilidae). Bulletin des Recherches Agronomiques de Gembloux, 12(4): 357-362.

ZANDEN, G. VAN DER, 1983. Taxonomische und faunistische Bemerkungen zu einigen Paläarktischen Bauchsammler-Arten (Insecta, Hymenoptera, Apoidea, Megachilidae). Faunistische Abhandlungen Staatliches Museum für Tierkunde in Dresden, [1982], 10(3): 124-139.

ZANDEN, G. VAN DER, 1986. Die paläarktischen Arten der Gattung Lithurgus Latreille, 1825 (Hymenoptera, Apoidea, Megachilidae). Mitteilungen aus dem Zoologischen Museum in Berlin, 62(1): 53-59.

ZANDEN, G. VAN DER, 1988. Nomenklatorische und taxonomische Bemerkungen zu einigen paläarktischen Arten der Familie Megachilidae (Insecta, Hymenoptera, Apoidea: Megachilidae). Reichenbachia, 26(10): 55-64.

ZANDEN, G. VAN DER, 1989. Neue oder wenig bekannte Arten und Unterarten der paläarktischen Megachiliden (Insecta, Hymenoptera, Apoidea, Megachilinae). Entomologische Abhandlungen Staatliches Museum für Tierkunde in Dresden, 53(6): 71-86.

ZANDEN, G. VAN DER, 1998. Neue paläarktischer Arten aus der Familie der Megachilidae (Insecta, Hymenoptera, Apoidea). Linzer Biologische Beiträge, 30(2): 523527.

PostsCRIPTUM: En este trabajo, como se indicó en los Agradecimientos, se ha seguido la indicación de uno de los evaluadores de poner en masculino los taxones específicos e infraespecíficos, haciéndolos coincidir con el género gramatical de Coelioxys. Sin embargo, tras haber conocido posteriormente las opiniones al respecto de otros especialistas en este grupo de himenópteros (Maximiliam Schwarz, Charles D. Michener y Fritz Gusenleitner), parece ser que la cuestión no está cerrada y debe ser investigada por la Comisión Internacional de Nomenclatura Zoológica. Téngase además en cuenta que Latreille, cuando describió el género Coelioxys, lo trató como femenino y tampoco Dalla Torre lo empleó en masculino más adelante, al igual que prácticamente todos los autores que, posteriormente, han descrito centenares de especies de este género.

Recibido, 7-II-2007 Aceptado, 31-V-2007 Publicado, 27-VI-2007 